\title{
Tableaux for Policy Synthesis for MDPs with PCTL* Constraints*
}

\author{
Peter Baumgartner, Sylvie Thiébaux, and Felipe Trevizan \\ Data61/CSIRO and Research School of Computer Science, ANU, Australia \\ Email: first.last@anu.edu.au
}

August 29, 2018

\begin{abstract}
Markov decision processes (MDPs) are the standard formalism for modelling sequential decision making in stochastic environments. Policy synthesis addresses the problem of how to control or limit the decisions an agent makes so that a given specification is met. In this paper we consider PCTL*, the probabilistic counterpart of CTL*, as the specification language. Because in general the policy synthesis problem for PCTL* is undecidable, we restrict to policies whose execution history memory is finitely bounded a priori. Surprisingly, no algorithm for policy synthesis for this natural and expressive framework has been developed so far. We close this gap and describe a tableau-based algorithm that, given an MDP and a PCTL* specification, derives in a non-deterministic way a system of (possibly nonlinear) equalities and inequalities. The solutions of this system, if any, describe the desired (stochastic) policies. Our main result in this paper is the correctness of our method, i.e., soundness, completeness and termination.
\end{abstract}

\section{Introduction}

Markov decision processes (MDPs) are the standard formalism for modelling sequential decision making in stochastic environments, where the effects of an agent's actions are only probabilistically known. The core problem is to synthesize a policy prescribing or restricting the actions that the agent may undertake, so as to guarantee that a given specification is met. Popular specification languages for this purpose include CTL, LTL, and their probabilistic counterparts PCTL and probabilistic LTL (pLTL). Traditional algorithms for policy synthesis and probabilistic temporal logic model-checking $[8,16]$ are based on bottom-up formula analysis $[14,15]$ or Rabin automata $[2,10,20]$.

*This is a longer version of the paper Peter Baumgartner, Sylvie Thiébaux, Felipe W. Trevizan: Tableaux for Policy Synthesis for MDPs with PCTL* Constraints. Proceedings of TABLEAUX 2017, pp. 175-192, Springer 2017, https://doi.org/10.1007/978-3-319-66902-1_11. It contains the proofs of the main results and it fixes a bug related to the definition of the semantics of PCTL* in terms of Markov chains. The semantics is now consistent with the standard semantics. It required adapting the calculus' P-rule to the new definition. 
We deviate from this mainstream research in two ways. The first significant deviation is that we consider PCTL* as a specification language, whereas previous synthesis approaches have been limited to pLTL and PCTL. PCTL* is the probabilistic counterpart of CTL* and subsumes both PCTL and pLTL. For example, the PCTL* formula $\mathbf{P}_{\geq 0.8} \mathbf{G}\left(\left(T>30^{\circ}\right) \rightarrow \mathbf{P}_{\geq 0.5} \mathbf{F} \mathbf{G}\left(T<24^{\circ}\right)\right)$ says "with probability at least 0.8 , whenever the temperature exceeds $30^{\circ}$ it will eventually stay below $24^{\circ}$ with probability at least $0.5^{\circ}$ ". Because of the nested probability operator $\mathbf{P}$ the formula is not in pLTL, and because of the nested temporal operators FG it is not in PCTL either.

Because in its full generality the policy synthesis problem for PCTL* is highly undecidable [4], one has to make concessions to obtain a decidable fragment. In this paper we chose to restrict to policies whose execution history memory is finitely bounded a priori. (For example, policies that choose actions in the current state dependent on the last ten preceding states.) However, we do target synthesizing stochastic policies, i.e., the actions are chosen according to a probability distribution (which generalizes the deterministic case and is known to be needed to satisfy certain formulas [2]). Surprisingly, no algorithm for policy synthesis in this somewhat restricted yet natural and expressive framework has been developed so far, and this paper closes this gap.

The second significant deviation from the mainstream is that we pursue a different approach based on analytic tableau and mathematical programming. Our tableau calculus is goal-oriented by focusing on the given PCTL* formula, which leads to analysing runs only on a by-need basis. This restricts the search space to partial policies that only cover the states reachable from the initial state under the policy and for which the formula imposes constraints on the actions that can be selected. In contrast, traditional automata based approaches require a full-blown state space exploration. (However, we do not have an implementation yet that allows us to evaluate the practical impact of this.) We also believe that our approach, although using somewhat nonstandard tableau features, is conceptually simpler and easier to comprehend. Of course, this is rather subjective.

On a high level, the algorithm works as follows. The input is an MDP, the finite-history component of the policy to be synthesized, and a PCTL* formula to be satisfied. Starting from the MDP's initial state, the tableau calculus symbolically executes the transition system given by the MDP by analysing the syntactic structure of given PCTL* formula, as usual with tableau calculi. Temporal formulas (e.g., FG-formulas) are expanded repeatedly using usual expansion laws and trigger state transitions. The process stops at trivial cases or when a certain loop condition is met. The underlying loop checking technique was developed only recently, by Mark Reynolds, in the context of tableau for satisfiability checking of LTL formulas [17]. It is an essential ingredient of our approach and we adapted it to our probabilistic setting.

Our tableaux have two kinds of branching. One kind is traditional or-branching, which represents non-deterministic choice by going down exactly one child node. It is used, e.g., in conjunction with recursively calling the tableau procedure itself. Such calls are necessary to deal with nested $\mathbf{P}$-operators, since at the time of analyzing a $\mathbf{P}$-formula it is, roughly speaking, unknown if the formula will hold true under the policy computed only later, as a result of the algorithm. The other kind of branching represents a union of alternatives. It is used for disjunctive formulas and for branching out from a state into successor states. Intuitively, computing the probability of a disjunctive formula $\phi_{1} \vee \phi_{2}$ is a function of the probabilities of 
both $\phi_{1}$ and $\phi_{2}$, so both need to be computed. Also, the probability of an $\mathbf{X}$-formula $\mathbf{X} \phi$ at a given state is a function of the probability of $\phi$ at all successor states, and so, again, all successor states need to be considered.

The tableau construction always terminates and derives a system of (possibly nonlinear) equalities and inequalities over the reals. The solutions of this system, if any, describe the desired stochastic, finite-history policies. The idea of representing policies as the solutions of a set of mathematical constraints is inspired by the abundant work in operations research, artificial intelligence, and robotics that optimally solves MDPs with simpler constraints using linear programming $[1,11,9,21]$.

Our main result in this paper is the correctness of our algorithm, i.e., soundness, completeness and termination. To our knowledge, it is the first and only policy synthesis algorithm for PCTL* that doesn't restrict the language (but only slightly the policies).

\section{Related Work}

Methods for solving the PCTL* model checking problem over Markov Chains are well established. The (general) policy synthesis however is harder than the model checking problem; it is known to be undecidable for even PCTL. The main procedure works bottom-up from the syntax tree of the given formula, akin to the standard CTL/CTL* model checking procedure. Embedded $\mathbf{P}$ formulas are recursively abstracted into boolean variables representing the sets of states satisfying these formulas, which are computed by LTL model checking techniques using Rabin automata. Our synthesis approach is rather different. While there is a rough correspondence in terms of recursive calls to treat $\mathbf{P}$ formulas, we do not need Rabin (or any other) automata; they are supplanted by the loop-check technique mentioned above.

The work the most closely related to ours is that of Brázdil et. al. [7, 5, 6]. Using Büchi automata, they obtain complexity results depending on the variant of the synthesis problem studied. However, they consider only qualitative fragments. For the case of interest in this paper, PCTL*, they obtain results for the fragment qPCTL*. The logic qPCTL* limits the use of the path quantifier $\mathbf{P}$ to formulas of the form $\mathbf{P}_{=1} \psi$ or $\mathbf{P}_{=0} \psi$, where $\psi$ is a path formula. On the other hand, we cover the full logic PCTL* which has arbitrary formulas of the form $\mathbf{P}_{\sim z} \psi$ where $\sim \in\{<, \leq,>, \geq\}$ and $z \in[0,1]$. In contrast to the works mentioned, we have to restrict to memory-dependent policies with an a priori limited finite memory. Otherwise the logic becomes highly undecidable [4].

\section{Preliminaries}

We assume the reader is familiar with basic concepts of Markov Decision Processes (MDPs), probabilistic model checking, and policy synthesis. See [16, 12, 3] for introductions and overviews. In the following we summarize the notions relevant to us and we introduce our notation.

Given a fixed finite vocabulary $A P$ of atomic propositions $a, b, c, \ldots$, a (propositional) interpretation $I$ is any subset of $A P$. It represents the assignment of each element in $I$ to true 
and each other atomic proposition in $A P \backslash I$ to false. A distribution on a countable set $X$ is a function $\mu: X \mapsto[0,1]$ such that $\sum_{x \in X} \mu(x)=1$, and $\operatorname{Dist}(X)$ is the set of all distributions on $X$.

A Markov Decision Process $(M D P)$ is a tuple $\mathcal{M}=\left(S, s_{\text {init }}, A, P, L\right)$ where: $S$ is a finite set of states; $s_{\text {init }} \in S$ is the initial state; $A$ is a finite set of actions and we denote by $A(s) \subseteq A$ the set of actions enabled in $s \in S ; P(t \mid s, \alpha)$ is the probability of transitioning to $t \in S$ after applying $\alpha \in A(s)$ in state $s$; and $L: S \mapsto 2^{A P}$ labels each state in $S$ with an interpretation. We assume that every state has at least one enabled action, i.e., $A(s) \neq \emptyset$ for all $s \in S$, and that $P$ is a distribution on enabled actions, i.e., $P(\cdot \mid s, \alpha) \in \operatorname{Dist}(S)$ iff $\alpha \in A(s)$ and $\Sigma_{t \in S} P(t \mid s, \alpha)=0$ iff $\alpha \notin A(s)$. For any $s$ and $\alpha \in A(s)$ let $\operatorname{Succ}(s, \alpha)=\{t \mid P(t \mid s, \alpha)>0\}$ be the states reachable from $s$ with non-zero probability after applying $\alpha$.

Given a state $s \in S$ of $\mathcal{M}$, a run from $s($ of $\mathcal{M})$ is an infinite sequence $r=\left(s=s_{1}\right) \stackrel{\alpha_{1}}{\longrightarrow}$ $s_{2} \stackrel{\alpha_{2}}{\longrightarrow} s_{3} \cdots$ of states $s_{i} \in S$ and actions $\alpha_{i} \in A\left(s_{i}\right)$ such that $P\left(s_{i+1} \mid s_{i}, \alpha_{i}\right)>0$, for all $i \geq 1$. We denote by Runs(s) the set of all runs from $s \in S$ and Runs $=\cup_{s \in S} R u n s(s)$. A path from $s \in S$ (of $\mathcal{M})$ is a finite prefix of a run from $s$ and we define Paths(s) and Paths in analogy to Runs(s) and Runs. We often write runs and paths in abbreviated form as state sequences $s_{1} s_{2} \cdots$ and leave the actions implicit. Given a path $p=s_{1} s_{2} \cdots s_{n}$ let first $(p)=s_{1}$ and last $(p)=s_{n}$. Similarly, for a run $r=s_{1} s_{2} \cdots$, first $(r)=s_{1}$ and $r[n]:=s_{n} s_{n+1} \cdots$, for any $n \geq 1$.

A policy $\pi$ represents a decision rule on how to choose an action given some information about the environment. In its most general form, a history-dependent (stochastic) policy (for $\mathcal{M})$ is a function $\pi$ : Paths $\mapsto \operatorname{Dist}(A)$ such that, for all $p \in$ Paths, $\pi(p)(\alpha)>0$ only if $\alpha \in A(\operatorname{last}(p))$. Technically, the MDP $\mathcal{M}$ together with $\pi$ induces an infinite-state Markov chain $\mathcal{M}_{\pi}=\left(S^{\pi}, s_{\text {init }}^{\pi}, A, P^{\pi}, L^{\pi}\right)$ over $S^{\pi}=$ Paths and this way provides a probability measure $\operatorname{Pr}^{M_{\pi}}$ on the set of runs of $\mathcal{M}$ under $\pi$. See [13,3] for details.

However, since Paths is an infinite set, a history-dependent policy might not be representable; moreover, the problem of finding such a policy that satisfies PCTL* constraints is undecidable [4]. We address these issues by working with finite-memory policies.

Finite-memory policies provide a distribution on actions for a current state from $S$ and a current mode. Formally, a finite-memory policy $($ for an $M D P \mathcal{M})$ is a DFA $\pi_{\text {fin }}=(M$, start, $\Delta$, act $)$ where $M$ is a finite set of modes, start: $S \mapsto M$ returns an initial mode to pair with a state $s \in S$, $\Delta: M \times S \mapsto M$ is the mode transition function, and act: $M \times S \mapsto \operatorname{Dist}(A)$ is the action probability function such that, for all $\langle m, s\rangle \in M \times S$, act $(m, s)(\alpha)>0$ only if $\alpha \in A(s)$. We abbreviate $\operatorname{act}(m, s)(\alpha)$ as $\operatorname{act}(m, s, \alpha)$.

Similarly to history-dependent policies above, a given $\mathcal{M}$ and $\pi_{\text {fin }}$ induce a Markov chain denoted as $\mathcal{M}_{\pi_{\text {fin }}}=\left(S^{\pi_{\text {fin }}}, s_{\text {init }}^{\pi_{\text {fin }}}, A, P^{\pi_{\text {fin }}}, L^{\pi_{\text {fin }}}\right)$. It has the finite state space $S^{\pi_{\text {fin }}}=M \times S$, initial state $s_{\text {init }}^{\pi_{\text {fin }}}=\left\langle\operatorname{start}\left(s_{\text {init }}\right), s_{\text {init }}\right\rangle$, set of actions $A$, transition probability function $P^{\pi_{\text {fin }}}\left(\left\langle m^{\prime}, s^{\prime}\right\rangle \mid\langle m, s\rangle\right):=$ $\Sigma_{\alpha \in A(s)} \operatorname{act}(m, s, \alpha) \cdot P\left(s^{\prime} \mid s, \alpha\right)$ if $m^{\prime}=\Delta(m, s)$ and 0 otherwise, and labelling function $L^{\pi_{\text {fin }}}(\langle m, s\rangle):=$ $L(s)$. A runfrom $s_{1}$ of $\mathcal{M}_{\pi_{\mathrm{fin}}}$ is a sequence of the form $\left\langle m_{1}, s_{1}\right\rangle\left\langle m_{2}, s_{2}\right\rangle \cdots$ such that $m_{1}=\operatorname{start}\left(s_{1}\right)$, $m_{i+1}=\Delta\left(m_{i}, s_{i}\right)$ and $P^{\pi_{\mathrm{fin}}}\left(\left\langle m_{i+1}, s_{i+1}\right\rangle \mid\left\langle m_{i}, s_{i}\right\rangle\right)>0$, for all $i \geq 1$. Notice that any run from $s_{1}$ of $\mathcal{M}_{\pi_{\text {fin }}}$ satisfies $\operatorname{act}\left(m_{i}, s_{i}, \alpha_{i}\right) \cdot P\left(s_{i+1} \mid s_{i}, \alpha_{i}\right)>0$ for some $\alpha_{i} \in A\left(s_{i}\right)$ and hence induces a run $s_{1} \stackrel{\alpha_{1}}{\longrightarrow} s_{2} \stackrel{\alpha_{2}}{\longrightarrow} s_{3} \cdots$ from $s_{1}$ of $\mathcal{M}$. The notions of Runs, Paths, first, last etc apply to Markov chains as well. For instance, $\operatorname{first}\left(\left\langle m_{1}, s_{1}\right\rangle\left\langle m_{2}, s_{2}\right\rangle \cdots\right)=\left\langle m_{1}, s_{1}\right\rangle$. Let $\operatorname{Pr}^{\mathcal{M}_{\pi_{\text {fin }}}}$ denote the probability measure on the set of runs of $\mathcal{M}_{\pi_{\mathrm{fin}}}$.

Finite-memory policies are more expressive than Markovian policies. For instance, the 
$\Delta$-component of $\pi_{\text {fin }}$ can be setup, e.g., to encode in $\langle m, s\rangle$ "the last ten states preceding $s$ ".

\subsection{Policy Synthesis for PCTL*}

(PCTL*) formulas follow the following grammar:

$$
\begin{aligned}
\phi & :=\text { true }|a \in A P| \phi \wedge \phi|\neg \phi| \mathbf{P}_{\sim z} \psi \\
\psi & :=\phi|\psi \wedge \psi| \neg \psi|\mathbf{X} \psi| \psi \mathbf{U} \psi
\end{aligned}
$$

(State formula)

(Path formula)

In the definition of state formulas, $\sim \in\{<, \leq,>, \geq\}$ and $0 \leq z \leq 1$. A proper path formula is a path formula that is not a state formula. A formula is classical iff it is made from atomic propositions and the Boolean connectives $\neg$ and $\wedge$ only (no occurrences of $\mathbf{P}, \mathbf{X}$ or $\mathbf{U}$ ). We write false as a shorthand for $\neg$ true.

We are going to define the semantics of PCTL* in terms of Markov chains in the usual way. That is, given an MDP $\mathcal{M}=\left(S, s_{\text {init }}, A, P, L\right)$ and a policy $\pi$, we fix the Markov chain $\mathcal{M}_{\pi}=\left(S^{\pi}, s_{\text {init }}^{\pi}, A, P^{\pi}, L^{\pi}\right)$ as described above. In fact, in our case the policies of interest will always be finite-memory policies.

Let Runs ${ }^{\mathcal{M}_{\pi}}$ denote the set of runs of $\mathcal{M}_{\pi}$. For state formulas $\phi$ and states $s \in S^{\pi}$ define the satisfaction relation $\mathcal{M}_{\pi}, s \mid=\phi$, briefly $s \mid=\phi$, as follows:

$$
\begin{aligned}
& s \mid=\text { true } \quad s \mid=\phi_{1} \wedge \phi_{2} \text { iff } s \mid=\phi_{1} \text { and } s \mid=\phi_{2} \\
& s \mid=a \text { iff } a \in L(s) \quad s \mid=\neg \phi \text { iff } s \mid \vDash \phi \\
& s \mid=\mathbf{P}_{\sim z} \psi \text { iff } \operatorname{Pr}^{\mathcal{M}_{\pi}}\left(\left\{r \in \text { Runs }^{\mathcal{M}_{\pi}}(s)\left|\mathcal{M}_{\pi}, r\right|=\psi\right\}\right) \sim z
\end{aligned}
$$

For path formulas $\psi$ and runs $r \in$ Runs $^{\mathcal{M}_{\pi}}$ define the satisfaction relation $\mathcal{M}_{\pi}, r \mid=\psi$, briefly $r=\psi$ as follows:

$$
\begin{array}{ll}
r=\phi \text { iff first }(r)=\phi & r=\psi_{1} \wedge \psi_{2} \text { iff } r \mid=\psi_{1} \text { and } r \mid=\psi_{2} \\
r=\neg \psi \text { iff } r \models \psi & r=\mathbf{X} \psi \text { iff } r[2] \models \psi \\
r=\psi_{1} \mathbf{U} \psi_{2} \text { iff exists } n \geq 1 \text { s.t. } r[n] \models \psi_{2} \text { and } r[m] \models \psi_{1} \text { for all } 1 \leq m<n
\end{array}
$$

We say that an MDP $\mathcal{M}$ and a policy $\pi$ satisfies $\phi$, written as $\mathcal{M}, \pi=\phi$ iff $\mathcal{M}_{\pi}, s_{\text {init }}^{\pi}=\phi$.

In this paper we focus on the problem of synthesizing only the act-component of an otherwise fully specified finite memory policy. More formally:

Definition 2.1 (Policy Synthesis Problem) Let $\mathcal{M}=\left(S, s_{\text {init }}, A, P, L\right)$ be an MDP, and $\pi_{\mathrm{fin}}=$ $(M$, start, $\Delta, \cdot)$ be a partially specified finite-memory policy with act unspecified. Given state formula $\phi$, find act s.th. $\mathcal{M}, \pi_{\text {fin }}=\phi$ if it exists, otherwise report failure.

\subsection{Useful Facts About PCTL* Operators}

Next we summarize some well-known or easy-to-prove facts about PCTL* operators. By the expansion laws for the $\mathbf{U}$-operator we mean the following equivalences:

$$
\psi_{1} \mathbf{U} \psi_{2} \equiv \psi_{2} \vee\left(\psi_{1} \wedge \mathbf{X}\left(\psi_{1} \mathbf{U} \psi_{2}\right)\right) \quad \neg\left(\psi_{1} \mathbf{U} \psi_{2}\right) \equiv \neg \psi_{2} \wedge\left(\neg \psi_{1} \vee \mathbf{X} \neg\left(\psi_{1} \mathbf{U} \psi_{2}\right)\right)
$$


For $\sim \in\{<, \leq,>, \geq\}$ define the operators $\approx$ and $[\sim]$ as follows:

$$
\overline{<}=\geq \quad \overline{\leq}=>\quad \overline{>}=\leq \quad \bar{z}=<\quad[<]=>\quad[\leq]=\geq \quad[>]=<\quad[\geq]=\leq
$$

Some of the following equivalences cannot be used for "model checking" PCTL* (the left (P1) equivalence, to be specific) where actions are implicitly universally quantified. In the context of Markov Chains, which we implicitly have, there is no problem:

$$
\begin{aligned}
\neg \mathbf{P}_{\sim z} \psi & \equiv \mathbf{P}_{\bar{\sim}_{z}} \psi \\
\mathbf{P}_{\geq 0} \psi & \equiv \text { true } \\
\mathbf{P}_{\leq 1} \psi & \equiv \text { true } \\
\mathbf{P}_{\geq u} \mathbf{P}_{\sim z} \psi & \equiv \mathbf{P}_{\sim z} \psi \quad \text { if } u \neq 0 \\
\mathbf{P}_{\leq u} \mathbf{P}_{\sim z} \psi & \equiv \mathbf{P}_{\geq 1-u} \mathbf{P}_{\bar{\sim}_{z}} \psi
\end{aligned}
$$

$$
\begin{aligned}
\mathbf{P}_{\sim z} \neg \psi & \equiv \mathbf{P}_{[\sim] 1-z} \psi \\
\mathbf{P}_{>1} \psi & \equiv \text { false } \\
\mathbf{P}_{<0} \psi & \equiv \text { false } \\
\mathbf{P}_{>u} \mathbf{P}_{\sim z} \psi & \equiv \mathbf{P}_{\sim z} \psi \quad \text { if } u \neq 1 \\
\mathbf{P}_{<u} \mathbf{P}_{\sim z} \psi & \equiv \mathbf{P}_{>1-u} \mathbf{P}_{\bar{\sim}} \psi
\end{aligned}
$$

\subsection{Nonlinear Programs}

Finally, a (nonlinear) program is a set $\Gamma$ of constraints of the form $e_{1} \bowtie e_{2}$ where $\bowtie \in\{<, \leq,>, \geq$ $, \doteq\}$ and $e_{1}$ and $e_{2}$ are arithmetic expressions comprised of numeric real constants and variables. The numeric operators are $\{+,-, \cdot, /\}$, all with their expected meaning (the symbol $\doteq$ is equality). All variables are implicitly bounded over the range $[0,1]$. A solver (for nonlinear programs) is a decision procedure that returns a satisfying variable assignment (a solution) for a given $\Gamma$, and reports unsatisfiability if no solution exists. We do not further discuss solvers in the rest of this paper, we just assume one as given. Examples of open source solvers include Ipopt and Couenne. ${ }^{1}$

\section{Tableau Calculus}

\subsection{Introduction and Overview}

We describe a tableau based algorithm for the policy synthesis problem in Definition 2.1. Hence assume as given an MDP $\mathcal{M}=\left(S, s_{\text {init }}, A, P, L\right)$ and a partially specified finite-memory policy $\pi_{\text {fin }}=(M$, start, $\Delta, \cdot)$ with act unspecified.

A labelled formula $\mathcal{F}$ is of the form $\langle m, s\rangle: \Psi$ where $\langle m, s\rangle \in M \times S$ and $\Psi$ is a possibly empty set of path formulas, interpreted conjunctively. When we speak of the probability of

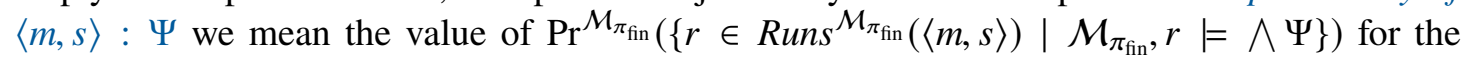
completed $\pi_{\text {fin }}$. For simplicity we also call $\Psi$ a "formula" and call $\langle m, s\rangle$ a policy state. A sequent is an expression of the form $\Gamma \vdash \mathcal{F}$ where $\Gamma$ is a program.

Our algorithm consists of three steps, the first one of which is a tableau construction. A tableau for $\Gamma \vdash \mathcal{F}$ is a finite tree whose root is labelled with $\Gamma \vdash \mathcal{F}$ and such that every inner node is labelled with the premise of an inference rule and its children are labelled with the conclusions, in order. If $\Gamma \vdash \mathcal{F}$ is the label of an inner node we call $\mathcal{F}$ the pivot of the node/sequent/inference. By a derivation from $\Gamma \vdash \mathcal{F}$, denoted by $\operatorname{Tableau}(\Gamma \vdash \mathcal{F})$, we mean any tableau for $\Gamma \vdash \mathcal{F}$

${ }^{1}$ http://projects. coin-or.org/. 
obtained by stepwise construction, starting from a root-node only tree and applying an inference rule to (the leaf of) every branch as long as possible. There is one inference rule, the $P$-rule, which recursively calls the algorithm itself. A branch is terminated when no inference rule is applicable, which is exactly the case when its leaf is labelled by a pseudo-sequent, detailed below. The inference rules can be applied in any way, subject to only preference constraints.

Given a state formula $\phi$, the algorithm starts with a derivation from $\Gamma_{\text {init }} \vdash \mathcal{F}_{\text {init }}:=$ $\left\{x_{\left\langle\text {start }\left(s_{\text {init }}\right), s_{\text {init }}\right\rangle} \doteq 1\right\} \vdash\left\langle\operatorname{start}\left(s_{\text {init }}\right), s_{\text {init }}\right\rangle:\{\phi\}$. (The constraint $\Gamma_{\text {init }}$ forces $\phi$ to be "true".) The derivation represents the obligation to derive a satisfiable extension $\Gamma_{\text {final }} \supseteq \Gamma_{\text {init }}$ whose solutions $\sigma$ determine the act-component act $\sigma$ of $\pi_{\text {fin }}$ such that $\mathcal{M}_{\pi_{\text {fin }}},\left\langle\operatorname{start}\left(s_{\text {init }}\right), s_{\text {init }}\right\rangle \mid=\phi$. In more detail, $\Gamma_{\text {final }}$ will contain constraints of the form $x_{\langle m, s\rangle}^{\alpha} \doteq 0$ or $x_{\langle m, s\rangle}^{\alpha}>0$ for the probability of applying action $\alpha$ in policy state $\langle m, s\rangle$. Let the policy domain of a program $\Gamma$ be the set of all policy states $\langle m, s\rangle \in M \times S$ such that $x_{\langle m, s\rangle}^{\alpha}$ occurs in $\Gamma$, for some $\alpha$. This lets us initially define $\operatorname{act}_{\sigma}(m, s, \alpha):=\sigma\left(x_{\langle m, s\rangle}^{\alpha}\right)$ for every $\langle m, s\rangle$ in the policy domain of $\Gamma_{\text {final }}$. Only for the purpose of satisfying the definition of finite memory policies, we then make act $\sigma$ trivially total by choosing an arbitrary distribution for $\operatorname{act}_{\sigma}(m, s)$ for all remaining $\langle m, s\rangle \in M \times S$. (The latter are not reachable and hence do not matter.) We call $\pi_{\text {fin }}(\sigma):=\left(M\right.$, start $_{,} \Delta$, act $\left.{ }_{\sigma}\right)$ the policy completed by $\sigma$.

Similarly, $\Gamma_{\text {final }}$ contains variables of the form $x_{\langle m, s\rangle}^{\Psi}$, and $\sigma\left(x_{\langle m, s\rangle}^{\Psi}\right)$ is the probability of $\langle m, s\rangle: \Psi$ under the policy $\pi_{\mathrm{fin}}(\sigma)$. (We actually need these variable indexed by tableau nodes, see below.) If $\Psi$ is a state formula its value will be 0 or 1 , encoding truth values.

Contrary to traditional tableau calculi, the result of the computation - the extension $\Gamma_{\text {final }}-$ cannot always be obtained in a branch-local way. To explain, there are two kinds of branching in our tableaux: don't-know (non-deterministic) branching and union branching. The former is always used for exhaustive case analysis, e.g., whether $x_{\langle m, s\rangle}^{\alpha} \doteq 0$ or $x_{\langle m, s\rangle}^{\alpha}>0$, and the algorithm guesses which alternative to take (cf. step 2 below). The latter analyzes the Boolean structure of the pivot. Unlike as with traditional tableaux, all children need to be expanded, and each fully expanded branch contributes to $\Gamma_{\text {final }}$.

More precisely, we formalize the synthesis algorithm as a three-step procedure. Step one consists in deriving Tableau $\left(\Gamma_{\text {init }} \vdash \mathcal{F}_{\text {init }}\right)$. Step two consists in removing from the step one tableau every don't-know branching by retaining exactly one child of the parent node of the don't-know branching, and deleting all other children and the subtrees below them. This itself is a don't-know non-deterministic process; it corresponds to going down one branch in traditional tableau. The result is denoted by $\operatorname{CHOose}\left(T_{1}\right)$, where $T_{1}$ is the step one tableau. Step three consists in first building a combined program by taking the union of the $\Gamma$ 's in the leaves of the branches of the step two tableau. This program then is extended with a set of constraints by the Force operator. More precisely, Forceing captures the situation when a run reaches a bottom strongly connected component (BSCC). Any formula is satisfied in a BSCC with probability 0 or 1 , which can be determined solely by qualitative formula evaluation in the BSCC. Details are below. For now let us just define GAmma $\left(T_{2}\right)=\bigcup\left\{\Gamma \mid \Gamma+\cdot\right.$ is the leaf of a branch in $\left.T_{2}\right\} \cup \operatorname{ForCE}\left(T_{2}\right)$ where $T_{2}=\operatorname{CHOose}\left(T_{1}\right)$.

Our main results are the following. See Appendix 7 for proofs.

Theorem 3.1 (Soundness) Let $\mathcal{M}=\left(S, s_{\text {init }}, A, P, L\right)$ be an $M D P, \pi_{\text {fin }}=(M, \operatorname{start}, \Delta, \cdot)$ be $a$ 
partially specified finite-memory policy with act unspecified, and $\phi$ a state formula. Suppose there is a program $\Gamma_{\text {final }}:=\operatorname{Gamma}\left(\operatorname{CHOOSE}\left(\operatorname{TableAU}\left(\left\{x_{\left\langle\text {start }\left(s_{\text {init }}\right), s_{\text {init }}\right\rangle} \doteq 1\right\} \vdash\left\langle\operatorname{start}\left(s_{\text {init }}\right), s_{\text {init }}\right\rangle\right.\right.\right.$ : $\{\phi\}))$ ) such that $\Gamma_{\text {final }}$ is satisfiable. Let $\sigma$ be any solution of $\Gamma_{\text {final }}$ and $\pi_{\mathrm{fin}}(\sigma)$ be the policy completed by $\sigma$. Then it holds $\mathcal{M}, \pi_{\mathrm{fin}}(\sigma) \mid=\phi$.

Theorem 3.2 (Completeness) Let $\mathcal{M}=\left(S, s_{\text {init }}, A, P, L\right)$ be an $M D P, \pi_{\text {fin }}=(M$, start, $\Delta$, act $) a$ finite-memory policy, and $\phi$ a state formula. Suppose $\mathcal{M}, \pi_{\mathrm{fin}}=\phi$. Then there is a satisfiable program $\Gamma_{\text {final }}:=\operatorname{Gamma}\left(\operatorname{Choose}\left(\operatorname{TableaU}\left(\left\{x_{\left\langle\operatorname{start}\left(s_{\text {init }}\right), s_{\text {init }}\right\rangle} \doteq 1\right\} \vdash\left\langle\operatorname{start}\left(s_{\text {init }}\right), s_{\text {init }}\right\rangle:\{\phi\}\right)\right)\right)$ and a solution $\sigma$ of $\Gamma_{\text {final }}$ such that $\operatorname{act}_{\sigma}(m, s, \alpha)=\operatorname{act}(m, s, \alpha)$ for every pair $\langle m, s\rangle$ in the policy domain of $\Gamma_{\text {final. }}$. Moreover $\mathcal{M}, \pi_{\mathrm{fin}}(\sigma) \models \phi$.

\subsection{Inference Rules}

There are two kinds of inference rules, giving two kinds of branching:

$$
\text { Name } \frac{\Gamma \vdash\langle m, s\rangle: \Psi}{\Gamma_{\text {left }} \vdash\langle m, s\rangle: \Psi \quad \Gamma_{\text {right }} \vdash\langle m, s\rangle: \Psi} \text { if condition }
$$

(Don't-know branching)

The pivot in the premise is always carried over into both conclusions. Only the constraint $\Gamma$ is modified into $\Gamma_{\text {left }} \supseteq \Gamma$ and $\Gamma_{\text {right }} \supseteq \Gamma$, respectively, for an exhaustive case analysis.

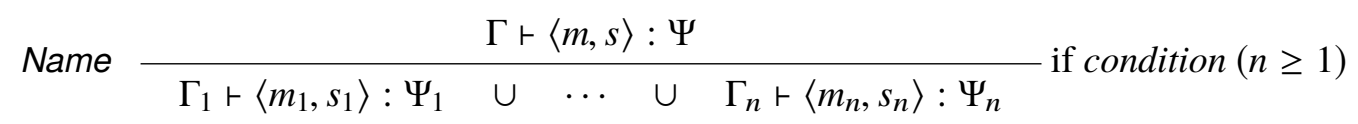

(Union branching)

All union branching rules satisfy $\Gamma_{i} \supseteq \Gamma$, and $\left\langle m_{i}, s_{i}\right\rangle=\langle m, s\rangle$ or $\left\langle m_{i}, s_{i}\right\rangle=\langle\Delta(m, s), t\rangle$ for some state $t$. The $\cup$-symbol is decoration for distinguishing the two kinds of branching but has no meaning beyond that. Union branching stands for the union of the runs from $\left\langle m_{i}, s_{i}\right\rangle$ satisfying $\Psi_{i}$, and computing its probability requires to develop all $n$ child nodes.

We need to clarify a technical add-on. Let $u$ be the tableau node with the premise pivot $\langle m, s\rangle: \Psi$. An union branching inference extends $u$ with children nodes, say, $u_{1}, \ldots, u_{n}$, with conclusion pivots $\left\langle m_{i}, s_{i}\right\rangle: \Psi_{i}$. The program $\Gamma_{n}$ will contain a constraint that makes a variable $\left(x_{u}\right)_{\langle m, s\rangle}^{\Psi}$ for the premise dependent on all variables $\left(x_{u_{i}}\right)_{\left\langle m_{i}, s_{i}\right\rangle}^{\left.\Psi_{i}\right\rangle}$ for the respective conclusions. This is a key invariant and is preserved by all inference rules. In order to lighten the notation, however, we usually drop the variable's index, leaving the node implicit. For instance, we write $x_{\langle m, s\rangle}^{\Psi}$ instead of $\left(x_{u}\right)_{\langle m, s\rangle}^{\Psi}$. The index $u$ is needed for not inadvertently identifying the same pivot at different points in the symbolic execution of a run. Fresh names $x, y, z, \ldots$ for the variables would do as well.

Most unary union branching rules have a premise $\Gamma \vdash\langle m, s\rangle:\{\psi\} \uplus \Psi$ and the conclusion is $\Gamma, \gamma_{\text {one }} \vdash\langle m, s\rangle: \Psi^{\prime}$, for some $\Psi^{\prime}$. The pivot is specified by pattern matching, where $\uplus$ is disjoint union, and $\gamma_{\text {one }}$ is a macro that expands to $x_{\langle m, s\rangle}^{\{\psi\} \uplus \Psi} \doteq x_{\langle m, s\rangle}^{\Psi^{\prime}}$. 
Other inference rules derive pseudo-sequents of the form $\Gamma \vdash \boldsymbol{X}, \Gamma \vdash \mathcal{V}, \Gamma \vdash$ Yes-Loop and $\Gamma \vdash$ No-Loop. They indicate that the probability of the pivot is 0,1 , or that a loop situation arises that may need further analysis. Pseudo-sequents are always leaves.

Now we turn to the concrete rules. They are listed in decreasing order of preference.

\section{Rules for classical formulas}

$$
\begin{aligned}
& \top \frac{\Gamma \vdash\langle m, s\rangle:\{\psi\} \uplus \Psi}{\Gamma, \gamma_{\text {one }} \vdash\langle m, s\rangle: \Psi}\left\{\begin{array} { l } 
{ \text { if } \psi \text { is clas- } } \\
{ \text { sical and } } \\
{ L ( s ) | = \psi }
\end{array} \quad x \frac { \Gamma \vdash \langle m , s \rangle : \{ \psi \} \uplus \Psi } { \Gamma , x _ { \langle m , s \rangle } ^ { \{ \psi \} \uplus \Psi } \doteq 0 \vdash \boldsymbol { X } } \left\{\begin{array}{l}
\text { if } \psi \text { is clas- } \\
\text { sical and } \\
L(s) \mid \psi \psi
\end{array}\right.\right. \\
& \checkmark \frac{\Gamma \vdash\langle m, s\rangle: \emptyset}{\Gamma, x_{\langle m, s\rangle}^{\emptyset} \doteq 1 \vdash \checkmark} \quad \neg \neg \frac{\Gamma \vdash\langle m, s\rangle:\{\neg \neg \psi\} \uplus \Psi}{\Gamma, \gamma_{\text {one }} \vdash\langle m, s\rangle:\{\psi\} \cup \Psi} \\
& \neg \mathbf{P} \frac{\Gamma \vdash\langle m, s\rangle:\left\{\neg \mathbf{P}_{\sim z} \psi\right\} \uplus \Psi}{\Gamma, \gamma_{\text {one }} \vdash\langle m, s\rangle:\left\{\mathbf{P}_{\sim_{z}} \psi\right\} \cup \Psi} \quad \mathbf{P}_{\neg} \frac{\Gamma \vdash\langle m, s\rangle:\left\{\mathbf{P}_{\sim z} \neg \psi\right\} \uplus \Psi}{\Gamma, \gamma_{\text {one }} \vdash\langle m, s\rangle:\left\{\mathbf{P}_{[\sim] 1-z} \psi\right\} \cup \Psi}
\end{aligned}
$$

These are rules for evaluating classical formulas and for negation. The $\boldsymbol{X}$ rule terminates the branch and assigns a probability of 0 to the premise pivot, as no run from $\langle m, s\rangle$ satisfies (the conjunction of) $\{\psi\} \uplus \Psi$, as $\psi$ is false in $s$. A similar reasoning applies to the $T$ and $\boldsymbol{V}$ rules. The $\neg \mathbf{P}$ and $\mathbf{P} \neg$ rules are justified by law (P1). The $\mathbf{P} \neg$ rule is needed for removing negation between $\mathbf{P}$-formulas as in $\mathbf{P}_{\sim z} \neg \mathbf{P}_{\sim v} \psi$.

\section{Rules for conjunctions}

$$
\begin{aligned}
& \wedge \frac{\Gamma \vdash\langle m, s\rangle:\left\{\psi_{1} \wedge \psi_{2}\right\} \uplus \Psi}{\Gamma, \gamma_{\text {one }} \vdash\langle m, s\rangle:\left\{\psi_{1}, \psi_{2}\right\} \cup \Psi} \\
& \neg \wedge \frac{\Gamma \vdash\langle m, s\rangle:\left\{\neg\left(\psi_{1} \wedge \psi_{2}\right)\right\} \uplus \Psi}{\Gamma \vdash\langle m, s\rangle:\left\{\neg \psi_{1}\right\} \cup \Psi \cup \quad \Gamma, \gamma \vdash\langle m, s\rangle:\left\{\psi_{1}, \neg \psi_{2}\right\} \cup \Psi} \\
& \text { where } \gamma=x_{\langle m, s\rangle}^{\left\{\neg\left(\psi_{1} \wedge \psi_{2}\right)\right\} \uplus \Psi} \doteq x_{\langle m, s\rangle}^{\left\{\neg \psi_{1}\right\} \cup \Psi}+x_{\langle m, s\rangle}^{\left\{\psi_{1}, \neg \psi_{2}\right\} \cup \Psi}
\end{aligned}
$$

These are rules for conjunction. Not both $\psi_{1}$ and $\psi_{2}$ can be classical by preference of the $T$ and $\boldsymbol{X}$ rules. The $\wedge$ rule is obvious with the conjunctive reading of formula sets. The $\neg \wedge$ rule deals, essentially, with the disjunction $\neg \psi_{1} \vee \neg \psi_{2}$, which requires splitting. However, unlike to the classical logic case, $\neg \psi_{1} \vee \neg \psi_{2}$ represents the union of the runs from $s$ satisfying $\neg \psi_{1}$ and the runs from $s$ satisfying $\neg \psi_{2}$. As these sets may overlap the rule works with a disjoint union by taking $\neg \psi_{1}$ on the one side, and $\psi_{1} \wedge \neg \psi_{2}$ on the other side so that it is correct to add their probabilities up in $\gamma$. 


\section{Rule for simplification of P-formulas}

$$
\begin{aligned}
& \mathbf{P} 1 \frac{\Gamma \vdash\langle m, s\rangle:\left\{\mathbf{P}_{\sim z} \psi\right\} \uplus \Psi}{\Gamma, \gamma_{\text {one }} \vdash\langle m, s\rangle:\left\{\psi^{\prime}\right\} \cup \Psi}\left\{\begin{array}{l}
\text { if } \mathbf{P}_{\sim z} \psi \text { is the left hand side of an equivalence } \\
(\mathrm{P} 2)-(\mathrm{P} 5) \text { and } \psi^{\prime} \text { is its right hand side }
\end{array}\right. \\
& \mathbf{P} 2 \frac{\Gamma \vdash\langle m, s\rangle:\left\{\mathbf{P}_{\sim z} \psi\right\} \uplus \Psi}{\Gamma, \gamma_{\text {one }} \vdash\langle m, s\rangle:\{\psi\} \cup \Psi} \text { if see text } \\
& \mathbf{P} 3 \frac{\Gamma \vdash\langle m, s\rangle:\left\{\mathbf{P}_{\sim z} \psi\right\} \uplus \Psi}{\Gamma, \gamma_{\text {one }} \vdash\langle m, s\rangle:\{\neg \psi\} \cup \Psi} \text { if see text }
\end{aligned}
$$

These are rules for simplifying $\mathbf{P}$-formulas. The condition in $\mathbf{P} 2$ is " $\sim \in\{>, \geq\}$ and $\psi$ is a state formula", and in P3 it is " $\sim \in\{<, \leq\}$ and $\psi$ is a state formula". In the rules P2 and P3 trivial cases for $z$ are excluded by preference of $\mathbf{P} 1$. Indeed, this preference is even needed for soundness.

The rule $\mathbf{P} 2$ can be explained as follows: suppose we want to know if $\mathcal{M}_{\pi_{\text {fin }}},\langle m, s\rangle \mid=\mathbf{P}_{\sim z} \psi$. For that we need the probability of the set of runs from $\langle m, s\rangle$ that satisfy $\psi$ and compare it with $z$. Because $\psi$ is a state formula this set is comprised of all runs from $s$ if $\mathcal{M}_{\pi_{\text {fin }}},\langle m, s\rangle \mid=\psi$, or the empty set otherwise, giving it probability 1 or 0 , respectively. With $\sim \in\{>, \geq\}$ conclude $\mathcal{M}_{\pi_{\text {fin }}},\langle m, s\rangle \mid=\mathbf{P}_{\sim z} \psi$, or its negation, respectively. The rule $\mathbf{P} 3$ is justified analogously. The only difference is that $\sim \in\{<, \leq\}$ and so the $\mathbf{P}_{\sim z}$ quantifier acts as a negation operator instead of idempotency.

At this stage, when all rules above have been applied exhaustively to a given branch, the leaf of that branch must be of the form $\Gamma \vdash\langle m, s\rangle:\left\{\mathbf{P}_{\sim z_{1}} \psi_{1}, \ldots, \mathbf{P}_{\sim z_{n}} \psi_{n}\right\}$, for some $n \geq 0$, where each $\psi_{i}$ is a non-negated proper path formula.

\section{Rules for P-formulas}

$$
\begin{aligned}
& \mathbf{P} \frac{\Gamma \vdash\langle m, s\rangle: \Psi}{\Gamma, \Gamma^{\prime}, \gamma_{\text {left }} \vdash\langle m, s\rangle: \Psi \quad \Gamma, \Gamma^{\prime}, \gamma_{\text {right }} \vdash\langle m, s\rangle: \Psi}\left\{\begin{array}{l}
\text { if } \mathbf{P}_{\sim z} \psi \in \Psi \text {, and } \\
\gamma_{\text {left }} \notin \Gamma \text { and } \gamma_{\text {right }} \notin \Gamma
\end{array}\right. \\
& \mathbf{P} \top \frac{\Gamma \vdash\langle m, s\rangle:\left\{\mathbf{P}_{\sim z} \psi\right\} \uplus \Psi}{\Gamma, \gamma_{\text {one }} \vdash\langle m, s\rangle: \Psi} \text { if } \gamma_{\text {left }} \in \Gamma \\
& \mathbf{P X} \frac{\Gamma \vdash\langle m, s\rangle:\left\{\mathbf{P}_{\sim z} \psi\right\} \uplus \Psi}{\Gamma, x_{\langle m, s\rangle}^{\left\langle\mathbf{P}_{\sim} \psi\right\} \uplus \Psi} \doteq 0 \vdash \boldsymbol{X}} \text { if } \gamma_{\text {right }} \in \Gamma \\
& \text { where } \Gamma^{\prime}=\operatorname{Gamma}(\operatorname{Choose}(\operatorname{Tableau}(\emptyset \vdash\langle m, s\rangle:\{\psi\}))) \text {, } \\
& \gamma_{\text {left }}=x_{\langle m, s\rangle}^{\{\psi\}} \sim z \text {, and } \gamma_{\text {right }}=x_{\langle m, s\rangle}^{\{\psi\}} \sim z
\end{aligned}
$$

Unlike classical formulas, $\mathbf{P}$-formulas cannot be evaluated in a state, because their truth value depends on the solution of the program $\Gamma_{\text {final }}$. The $\mathbf{P}$ rule analyzes $\mathbf{P}_{\sim z} \psi$ in a deferred way by first getting a constraint $x_{\langle m, s\rangle}^{\{\psi\}} \doteq e$, for some expression $e$, for the probability of $\langle m, s\rangle:\{\psi\}$ by a recursive call. This call is not needed if $\Gamma$ already determines a truth value for $\mathbf{P}_{\sim z} \psi$ because 
of $\gamma_{\text {left }} \in \Gamma$ or $\gamma_{\text {right }} \in \Gamma$. These tests are done modulo node labels of variables, i.e., $\left(x_{u}\right)_{\langle m, s\rangle}^{\{\psi\}}$ and $\left(x_{v}\right)_{\langle m, s\rangle}^{\{\psi\}}$ are considered equal for any $u, v$. Because the value of $e$ is not known at the time of the inference, the $\mathbf{P}$ rule don't-know non-deterministically branches out into whether $x_{\langle m, s\rangle}^{\{\psi\}} \sim z$ holds or not, as per the constraints $\gamma_{\text {left }}$ and $\gamma_{\text {right }}$. The $\mathbf{P T}$ and $\mathbf{P X}$ rules then lift the corresponding case to the evaluation of $\mathbf{P}_{\sim z} \psi$, which is possible now thanks to $\gamma_{\text {left }}$ or $\gamma_{\text {right }}$.

Observe the analogy between these rules and their counterparts $T$ and $\boldsymbol{X}$ for classical formulas. Note that the rules $\mathbf{P}, \mathbf{P} T$ and $\mathbf{P X}$ cannot be combined into one, because $\gamma_{\text {left }}$ or $\gamma_{\text {right }}$ could have been added earlier, further above in the branch, or in a recursive call. In this case only $\mathbf{P T / P X}$ can applied.

At this stage, in a leaf $\Gamma \vdash\langle m, s\rangle: \Psi$ the set $\Psi$ cannot contain any state formulas, as they would all be eliminated by the inference rules above; all formulas in $\Psi$ now are possibly negated $\mathbf{X}$-formulas or $\mathbf{U}$-formulas.

\section{Rules for U-formulas}

$$
\begin{gathered}
\mathbf{U} \frac{\Gamma \vdash\langle m, s\rangle:\left\{\psi_{1} \mathbf{U} \psi_{2}\right\} \uplus \Psi}{\Gamma \vdash\langle m, s\rangle:\left\{\psi_{2}\right\} \cup \Psi \quad \cup \quad \Gamma, \gamma \vdash\langle m, s\rangle:\left\{\psi_{1}, \neg \psi_{2}, \mathbf{X}\left(\psi_{1} \mathbf{U} \psi_{2}\right)\right\} \cup \Psi} \\
\text { where } \gamma=x_{\langle m, s\rangle}^{\left\{\psi_{1} \mathbf{U} \psi_{2}\right\} \uplus \Psi} \doteq x_{\langle m, s\rangle}^{\left\{\psi_{2}\right\} \cup \Psi}+x_{\langle m, s\rangle}^{\left\{\psi_{1}, \neg \psi_{2}, \mathbf{X}\left(\psi_{1} \mathbf{U} \psi_{2}\right)\right\} \cup \Psi} \\
\neg \begin{array}{c}
\Gamma \vdash\langle m, s\rangle:\left\{\neg\left(\psi_{1} \mathbf{U} \psi_{2}\right)\right\} \uplus \Psi \\
\text { where } \gamma=x_{\langle m, s\rangle}^{\left\{\neg\left(\psi_{1} \mathbf{U} \psi_{2}\right)\right\} \uplus \Psi} \doteq x_{\langle m, s\rangle}^{\left\{\neg \psi_{1}, \neg \psi_{2}\right\} \cup \Psi}+x_{\langle m, s\rangle}^{\left\{\psi_{1}, \neg \psi_{2}, \mathbf{X} \neg\left(\psi_{1} \mathbf{U} \psi_{2}\right)\right\} \cup \Psi}
\end{array}
\end{gathered}
$$

These are expansion rules for $\mathbf{U}$-formulas. The standard expansion law is $\psi_{1} \mathbf{U} \psi_{2} \equiv \psi_{2} \vee\left(\psi_{1} \wedge\right.$ $\left.\mathbf{X}\left(\psi_{1} \mathbf{U} \psi_{2}\right)\right)$. As with the $\neg \wedge$ rule, the disjunction in the expanded formula needs to be disjoint by taking $\psi_{2} \vee\left(\psi_{1} \wedge \neg \psi_{2} \wedge \mathbf{X}\left(\psi_{1} \mathbf{U} \psi_{2}\right)\right)$ instead. Similarly for $\neg \mathbf{U}$.

\section{Rule for $\neg X$}

$$
\neg \mathbf{X} \frac{\Gamma \vdash\langle m, s\rangle:\{\neg \mathbf{X} \psi\} \uplus \Psi}{\Gamma, \gamma_{\text {one }} \vdash\langle m, s\rangle:\{\mathbf{X} \neg \psi\} \cup \Psi}
$$

The $\neg \mathbf{X}$ rule is obvious.

At this stage, if $\Gamma \vdash\langle m, s\rangle: \Psi$ is a leaf sequent then $\Psi$ is of the form $\left\{\mathbf{X} \psi_{1}, \ldots, \mathbf{X} \psi_{n}\right\}$, for some $n \geq 1$. This is an important configuration that justifies a name: we say that a labelled formula $\langle m, s\rangle: \Psi$, a sequent $\Gamma \vdash\langle m, s\rangle: \Psi$ or a node labelled with $\Gamma \vdash\langle m, s\rangle: \Psi$ is poised if $\Psi$ is of the form $\left\{\mathbf{X} \psi_{1}, \ldots, \mathbf{X} \psi_{n}\right\}$ where $n \geq 1$. (The notion "poised" is taken from [17].) A poised $\langle m, s\rangle:\left\{\mathbf{X} \psi_{1}, \ldots, \mathbf{X} \psi_{n}\right\}$ will be expanded by transition into the successor states of $s$ by using enabled actions $\alpha \in A(s)$. That some $\alpha$ is enabled does not, however, preclude a policy with $\operatorname{act}_{\sigma}(m, s, \alpha)=0$. The rule A makes a guess whether this is the case or not: 


\section{Rules for prescribing actions}

$$
\begin{aligned}
& \text { A } \frac{\Gamma \vdash\langle m, s\rangle: \Psi}{\Gamma, \gamma_{\text {left }} \vdash\langle m, s\rangle: \Psi \quad \Gamma, \gamma_{\text {right }} \vdash\langle m, s\rangle: \Psi}\left\{\begin{array}{l}
\text { if } \Gamma \vdash\langle m, s\rangle: \Psi \text { is poised, } \\
\alpha \in A(s), \gamma_{\text {left }} \notin \Gamma \text { and } \gamma_{\text {right }} \notin \Gamma
\end{array}\right. \\
& \text { where } \gamma_{\text {left }}=x_{\langle m, s\rangle}^{\alpha} \doteq 0 \text { and } \gamma_{\text {right }}=x_{\langle m, s\rangle}^{\alpha}>0
\end{aligned}
$$

With a minor modification we get a calculus for deterministic policies. It only requires to re-define $\gamma_{\text {right }}$ as $\gamma_{\text {right }}=x_{\langle m, s\rangle}^{\alpha} \doteq 1$. As a benefit the program $\Gamma_{\text {final }}$ will be linear.

After the $\mathrm{A}$ rule has been applied exhaustively, for each $\alpha \in A(s)$ either $x_{\langle m, s\rangle}^{\alpha}>0 \in \Gamma$ or $x_{\langle m, s\rangle}^{\alpha} \doteq 0 \in \Gamma$. If $x_{\langle m, s\rangle}^{\alpha}>0 \in \Gamma$ we say that $\alpha$ is prescribed in $\langle m, s\rangle$ by $\Gamma$ and define Prescribed $(\langle m, s\rangle, \Gamma)=\left\{\alpha \mid x_{\langle m, s\rangle}^{\alpha}>0 \in \Gamma\right\}$.

The set of prescribed actions in a policy state determines the Succ-relation of the Markov chain under construction. To get the required distribution over enabled actions, it suffices to enforce a distribution over prescribed actions, with this inference rule:

$$
\begin{aligned}
& \text { Prescribed } \frac{\Gamma \vdash\langle m, s\rangle: \Psi}{\Gamma, \gamma_{\langle m, s\rangle}^{\alpha} \vdash\langle m, s\rangle: \Psi}\left\{\begin{array}{l}
\text { if } \Gamma \vdash\langle m, s\rangle: \Psi \text { is poised, } \\
\alpha \in A(s) \text { and } \gamma_{\langle m, s\rangle}^{\alpha} \notin \Gamma
\end{array}\right. \\
& \text { where } \gamma_{\langle m, s\rangle}^{\alpha}=\Sigma_{\alpha \in \operatorname{Prescribed}(\langle m, s\rangle, \Gamma)} x_{\langle m, s\rangle}^{\alpha} \doteq 1
\end{aligned}
$$

If the CHoose operator in step two selects the leftmost branch among the A-inferences then $\Gamma_{\text {final }}$ contains $x_{\langle m, s\rangle}^{\alpha} \doteq 0$, for all $\alpha \in A(s)$. This is inconsistent with the constraint introduced by the Prescribed-inference, corresponding to the fact that runs containing $\langle m, s\rangle$ in this case do not exist.

\section{Blocking}

We are now turning to a "loop check" which is essential for termination, by, essentially, blocking the expansion of certain states into successor states that do not mark progress. For that, we need some more concepts. For two nodes $u$ and $v$ in a branch we say that $u$ is an ancestor of $v$ and write $u \leq v$ if $u=v$ or $u$ is closer to the root than $v$. An ancestor is proper, written as $u<v$, if $u \leq v$ but $u \neq v$. We say that two sequents $\Gamma_{1} \vdash \mathcal{F}_{1}$ and $\Gamma_{2} \vdash \mathcal{F}_{2}$ are indistinguishable iff $\mathcal{F}_{1}=\mathcal{F}_{2}$, i.e., they differ only in their $\Gamma$-components. Two nodes $u$ and $v$ are indistinguishable iff their sequents are. We write $\Psi_{u}$ to denote the formula component of $u$ 's label, i.e., to say that the label is of the form $\Gamma \vdash\langle m, s\rangle: \Psi_{u}$; similarly for $\mathcal{F}_{u}$ to denote $u$ 's labelled formula.

Definition 3.3 (Blocking) Let $w$ be a poised leaf and $v<w$ an ancestor node. If (i) $v$ and $w$ are indistinguishable, and (ii) for every $\mathbf{X}$-eventuality $\mathbf{X}\left(\psi_{1} \mathbf{U} \psi_{2}\right)$ in $\Psi_{v}$ there is a node $x$ with $v<x \leq w$ such that $\psi_{2} \in \Psi_{x}$ then $w$ is yes-blocked by $v$. If there is an ancestor node $u<v$ such that (i) $u$ is indistinguishable from $v$ and $v$ is indistinguishable from $w$ (and hence $u$ is indistinguishable from $w$ ), and (ii) for every $\mathbf{X}$-eventuality $\mathbf{X}\left(\psi_{1} \mathbf{U} \psi_{2}\right)$ in $\Psi_{u}$, if there is a node $x$ with $\psi_{2} \in \Psi_{x}$ and $v<x \leq w$ then there is a node $y$ with $\psi_{2} \in \Psi_{y}$ and $u<y \leq v$, then $w$ is no-blocked by $u$.

When we say that a sequent is yes/no-blocked we mean that its node is yes/no-blocked. 
In the yes-blocking case all $\mathbf{X}$-eventualities in $\Psi_{v}$ become satisfied along the way from $v$ to $w$. This is why $w$ represents a success case. In the no-blocking case some $\mathbf{X}$-eventualities in $\Psi_{v}$ may have been satisfied along the way from $u$ to $v$, but not all, as this would be a yes-blocking instead. Moreover, no progress has been made along the way from $v$ to $w$ for satisfying the missing $\mathbf{X}$-eventualities. This is why $w$ represents a failure case. The blocking scheme is adapted from [17] for LTL satisfiability to our probabilistic case. See [18, 17] for more explanations and examples, which are instructive also for its usage in our framework.

Blocking is used in the following inference rules, collectively called the Loop rules. The node $v$ there is an ancestor node of the leaf the rule is applied to.

$$
\begin{array}{cc}
\text { Yes-Loop } \frac{\Gamma \vdash\langle m, s\rangle: \Psi}{\Gamma, x_{\langle m, s\rangle}^{\Psi} \doteq\left(x_{v}\right)_{\langle m, s\rangle}^{\Psi} \vdash \text { Yes-Loop }} \text { if } \Gamma \vdash\langle m, s\rangle: \Psi \text { is yes-blocked by } v \\
\text { No-Loop } \frac{\Gamma \vdash\langle m, s\rangle: \Psi}{\Gamma, x_{\langle m, s\rangle}^{\Psi} \doteq\left(x_{v}\right)_{\langle m, s\rangle}^{\Psi} \vdash \text { No-Loop }} \text { if } \Gamma \vdash\langle m, s\rangle: \Psi \text { is no-blocked by } v
\end{array}
$$

In either case, if $v$ is indistinguishable from $w$ then the probability of $\mathcal{F}_{v}$ and $\mathcal{F}_{w}$ are exactly the same, just because $\mathcal{F}_{v}=\mathcal{F}_{w}$. This justifies adding $x_{\langle m, s\rangle}^{\Psi} \doteq\left(x_{v}\right)_{\langle m, s\rangle}^{\Psi}$.

The Loop rules have a side-effect that we do not formalize: they add a link from the conclusion node (the new leaf node) to the blocking node $v$, called the backlink. It turns the tableau into a graph that is no longer a tree. The backlinks are used only for reachability analysis in step three of the algorithm. Figure 1 has a graphical depiction.

By preference of inference rules, the $\mathbf{X}$ rule introduced next can be applied only if a Loop rule does not apply. The Loop rules are at the core of the termination argument.

This argument is standard for calculi based on formula expansion, as embodied in the $\mathbf{U}$ and $\neg \boldsymbol{U}$ rules: the sets of formulas obtainable by these rules is a subset of an a priori determined finite set of formulas. This set consists of all subformulas of the given formula closed under negation and other operators. Any infinite branch hence would have to repeat one of these sets infinitely often, which is impossible with the loop rules. Moreover, the state set $S$ and the mode set $M$ are finite and so the other rules do not cause problems either.

For economy of notation, when $\Psi=\left\{\psi_{1}, \ldots, \psi_{n}\right\}$, for some $\psi_{1}, \ldots, \psi_{n}$ and $n>0$, let $\mathbf{X} \Psi$ denote the set $\left\{\mathbf{X} \psi_{1}, \ldots, \mathbf{X} \psi_{n}\right\}$.

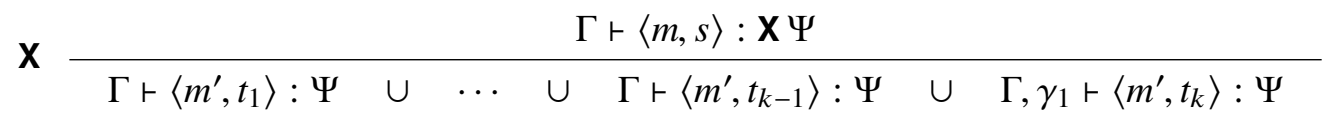

$$
\begin{aligned}
& \text { where } \quad m^{\prime}=\Delta(m, s) \\
& \left\{t_{1}, \ldots, t_{k}\right\}=\bigcup_{\alpha \in \operatorname{Prescribed}(\langle m, s\rangle, \Gamma)} \operatorname{Succ}(s, \alpha) \text {, for some } k \geq 0 \\
& \gamma_{1}=x_{\langle m, s\rangle}^{\mathbf{X} \Psi} \doteq \Sigma_{\alpha \in \operatorname{Prescribed}(\langle m, s\rangle, \Gamma)}\left[x_{\langle m, s\rangle}^{\alpha} \cdot\left(\Sigma_{t \in \operatorname{Succ}(s, \alpha)} P(t \mid s, \alpha) \cdot x_{\left\langle m^{\prime}, t\right\rangle}^{\Psi}\right)\right]
\end{aligned}
$$

This is the (only) rule for expansion into successor states.

If $u$ is the node the $\mathbf{X}$ rule is applied to and $u_{1}, \ldots, u_{k}$ are its children then each $u_{i}$ is called an $\mathbf{X}$-successor (of $u$ ). 
The $\mathbf{X}$ rule follows the set of actions prescribed in $\langle m, s\rangle$ by $\Gamma$ through to successor states. This requires summing up the probabilities of carrying out $\alpha$, as represented by $x_{\langle m, s\rangle}^{\alpha}$, multiplied by the sums of the successor probabilities weighted by the respective transition probabilities. This is expressed in the constraint $\gamma_{1}$. Only these $k$ successors need to be summed up, as all other, non-prescribed successors, have probability 0 .

\subsection{Forcing Probabilities}

We are now turning to the FORCE operator which we left open in step three of the algorithm. It forces a probability 0 or 1 for certain labelled formulas occurring in a bottom strongly connected component in a tree from step two. The tree in the figure to the right helps to illustrate the concepts introduced in the following.

We need some basic notions from graph theory. A subset $M$ of the nodes $N$ of a given graph is strongly connected if, for each pair of nodes $u$ and $v$ in $M, v$ is reachable from $u$ passing only through states in $M$. A strongly connected component (SCC) is a maximally strongly connected set of nodes (i.e., no superset of it is also strongly connected). A bottom strongly connected component (BSCC) is a SCC $M$ from which no state outside $M$ is reachable from $M$.

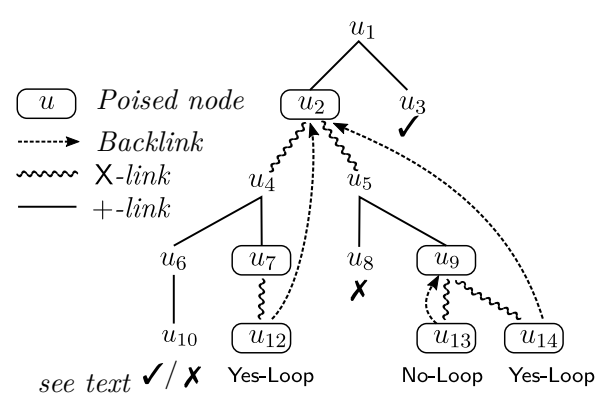

Figure 1: An example tableau $T$ from step 2. The subgraph below $u_{2}$ is a strongly connected component if $u_{10}$ is $\boldsymbol{X}$-ed.

Let $T=\operatorname{CHOOSE}(\mathrm{TABLEAU}(\Gamma \vdash \mathcal{F}))$ be a tree without don't-know branching obtained in step 2 . We wish to take $T$ together with its backlinks as the graph under consideration and analyse its BSCCs. However, for doing so we cannot take $T$ as it is. On the one hand, our tableaux describe state transitions introduced by $\mathbf{X}$ rule applications. Intuitively, these are amenable to BSCC analysis as one would do for state transition systems. On the other hand, $T$ has interspersed rule applications for analysing Boolean structure, which distort the state transition structure. These rule applications have to be taken into account prior to the BSCC analysis proper.

For this, we distinguish between $\mathbf{X}$-links and +-links in $T$. An $\mathbf{X}$-link is an edge between a node and its child if the $\mathbf{X}$ rule was applied to the node, making its child an $\mathbf{X}$-successor, otherwise it is a +-link. ("+-link" because probabilities are summed up.)

Let $u$ be a node in $T$ and $\operatorname{Subtree}_{T}(u)$, or just Subtree $(u)$, the subtree of $T$ rooted at $u$ without the backlinks. We say that $u$ is a 0 -deadend (in $T$ ) if $\operatorname{Subtree}_{T}(u)$ has no $\mathbf{X}$-links and every leaf in $\operatorname{Subtree}_{T}(u)$ is $\boldsymbol{X}$-ed. In a 0 -deadend the probabilities all add up to a zero probability for the pivot of $u$. This is shown by an easy inductive argument.

Definition 3.4 (Ambiguous node) Let $u$ be a node in $T$. We say that $u$ is ambiguous (in $T$ ) iff (i) $\operatorname{Subtree}_{T}(u)$ contains no $\checkmark$-ed leaf, and (ii) $\operatorname{Subtree}_{T}(u)$ contains no $\mathbf{X}$-successor 0-deadend node. We say that $u$ is unambiguous iff $u$ is not ambiguous.

The main application of Definition 3.4 is when the node $u$ is the root of a BSCCs, defined below. The probability of $u$ 's pivot $\langle m, s\rangle: \Psi$ then is not uniquely determined. This is because 
expanding $u$ always leads to a cycle, a node with the same pivot, and there is no escape from that according to conditions (i) or (ii) in Definition 3.4. In other words, the probability of $\langle m, s\rangle: \Psi$ is defined only in terms of itself. ${ }^{2}$

In the figure above, the node $u_{1}$ is unambiguous because of case (i) in Definition 3.4. Assuming $u_{10}$ is $\checkmark$-ed, the node $u_{2}$ is unambiguous by case (i). The pivot in $u_{10}$, then, has probability 1 which is propagated upwards to $u_{4}$ (and enforces probability 0 for the pivot of $u_{7}$ ). It contributes a non-zero probability to the transition from $u_{2}$ to $u_{4}$ and this way escapes a cycle. If $u_{10}$ is $\boldsymbol{X}$-ed, the node $u_{2}$ is ambiguous.

If case (ii) in Definition 3.4 is violated there is an $\mathbf{X}$-successor node whose pivot has probability 0 . Because every $\mathbf{X}$-link has a non-zero transition probability, the probabilities obtained through the other $\mathbf{X}$-successor nodes add up to a value strictly less than 1 . This also escapes the cycle leading to underspecified programs (not illustrated above).

Let $O(T)=\{w \mid w$ is a node in some 0 -deadend of $T\}$ be all nodes in all 0-deadends in $T$. In the example, $0(T)=\left\{u_{6}, u_{10}, u_{8}\right\}$ if $u_{10}$ is $\boldsymbol{X}$-ed and $0(T)=\left\{u_{8}\right\}$ if $u_{10}$ is $\boldsymbol{J}$-ed.

Let $u$ be a node in $T$ and $M(u)=\{w \mid w$ is a node in Subtree $(u)\} \backslash 0(T)$. That is, $M(u)$ consists of the nodes in the subtree rooted at $u$ after ignoring the nodes from the 0-deadend subtrees. In the example $M\left(u_{2}\right)=\left\{u_{2}, u_{4}, u_{5}, u_{7}, u_{9}, u_{12}, u_{13}, u_{14}\right\}$ if $u_{10}$ is $\boldsymbol{X}$-ed. If $u_{10}$ is $\boldsymbol{J}$-ed then $u_{6}$ and $u_{10}$ have to be added.

We say that $u$ is the root of a BSCC (in T) iff $u$ is poised, ambiguous and $M(u)$ is a BSCC in $T$ (together with the backlinks). In the example, assume that $u_{10}$ is $\boldsymbol{X}$-ed. Then $u_{2}$ is poised, ambiguous and the root of a BSCC. In the example, that $M\left(u_{2}\right)$ is a BSCC in $T$ is easy to verify.

Now suppose that $u$ is the root of a BSCC with pivot $\langle m, s\rangle: \mathbf{X} \Psi$. This means that the probability of $\langle m, s\rangle: \mathbf{X} \Psi$ is not uniquely determined. This situation then is fixed by means of the FORCE operation, generally defined as follows:

$$
\begin{gathered}
\operatorname{BscC}(T):=\{u \mid u \text { is the root of a BSCC in } T\} \\
\operatorname{Force}(T):=\left\{\left(x_{u}\right)_{\langle m, s\rangle}^{\mathbf{X}} \doteq \chi \mid u \in \operatorname{BscC}(T),\right. \text { and } \\
\text { if some leaf of the subtree rooted at } u \text { is a Yes-Loop } \\
\text { then } \chi=1 \text { else } \chi=0\}
\end{gathered}
$$

That is, Forceing removes the ambiguity for the probability of the pivot $\langle m, s\rangle: \mathbf{X} \Psi$ at the root $u$ of a BSCC by setting it to 1 or to 0 . If Forceing adds $\left(x_{u}\right)_{\langle m, s\rangle}^{\mathbf{X} \Psi} \doteq 1$ then there is a run that satisfies every $\mathbf{X}$-eventuality in $\mathbf{X} \Psi$, by following the branch to a Yes-Loop. Because we are looking at a BSCC, for fairness reasons, every run will do this, and infinitely often so, this way giving $\mathbf{X} \Psi$ probability 1 . Otherwise, if there is no Yes-Loop, there is some $\mathbf{X}$-eventuality in $\mathbf{X} \Psi$ that cannot be satisfied, forcing probability 0 .

\section{Conclusions and Future Work}

In this paper we presented a first-of-its kind algorithm for the controller synthesis problem for Markov Decision Processes whose intended behavior is described by PCTL* formulas. The only

\footnotetext{
${ }^{2}$ In terms of the resulting program, $\left(x_{u}\right)_{\langle m, s\rangle}^{\psi}$ is not constrained to any specific value in [0..1]. This can be shown by "substituting in" the equalities in $\Gamma_{\text {final }}$ for the probabilities of the pivots in the subtrees below $u$ and arithmetic simplifications.
} 
restriction we had to make - to get decidability - is to require policies with finite history. We like to propose that the description of the algorithm is material enough for one paper, and so we leave many interesting questions for future work.

The most pressing theoretical question concerns the exact worst-case complexity of the algorithm. Related to that, it will be interesting to specialize and analyze our framework for fragments of PCTL*, such as probabilistic LTL and CTL or simpler fragments and restricted classes of policies that might lead to linear programs (and ideally to solving only a polynomial number of such programs). For instance, we already mentioned that computing deterministic policies leads to linear programs in our tableau (see the description of the A inference rule how this is done.) Moreover, it is well-known that cost-optimal stochastic policies for classes of MDPs with simple constraints bounding the probability of reaching a goal state can be synthesized in linear time in the size of the MDP by solving a single linear program $[1,11]$. An interesting question is how far these simple constraints can be generalised towards PCTL* whilst remaining in the linear programming framework (see e.g. [19]).

On implementation, a naïve implementation of the algorithm as presented above would perform poorly in practice. However, it is easy to exploit some straightforward observations for better performance. For instance, steps one (tableau construction) and two (committing to a don't-know non-deterministic choice) should be combined into one. Then, if a don't know non-deterministic inference rule is carried out the first time, every subsequent inference with the same rule and pivot can be forced to the same conclusion, at the time the rule is applied. Otherwise an inconsistent program would result, which never needs to be searched for. Regarding space, although all children of a union branching inference rule need to be expanded, this does not imply they always all need to be kept in memory simultaneously. Nodes can be expanded in a one-branch-at-a-time fashion and using a global variable for $\Gamma_{\text {final }}$ for collecting the programs in the leaves of the branches if they do not belong to a bottom strongly connected component. Otherwise, the situation is less obvious and we leave it to future work. Another good source of efficiency improvements comes from more traditional tableau. It will be mandatory to exploit techniques such as dependency-directed backtracking, lemma learning, and early failure checking for search space pruning.

\section{Acknowledgements}

This research was funded by AFOSR grant FA2386-15-1-4015. We would also like to thank the anonymous reviewers for their constructive and helpful comments.

\section{Additional Operators and Useful Equivalences}

Additional operators can be defined on top of the temporal operator $\mathbf{U}$, as usual. In particular, false $:=\neg$ true and

$$
\begin{aligned}
& \mathbf{F} \psi:=\text { true } \mathbf{U} \psi \\
& \mathbf{G} \psi:=\neg \mathbf{F} \neg \psi \quad(\equiv \text { false } \mathbf{R} \psi) \\
& \psi_{1} \mathbf{R} \psi_{2}:=\neg\left(\neg \psi_{1} \mathbf{U} \neg \psi_{2}\right) \\
& \psi_{1} \mathbf{W} \psi_{2}:=\left(\psi_{1} \mathbf{U} \psi_{2}\right) \vee \mathbf{G} \psi_{1} \quad\left(\equiv \psi_{2} \mathbf{R}\left(\psi_{2} \vee \psi_{1}\right)\right)
\end{aligned}
$$


For the "release" operator $\mathbf{R}$, the formula $\psi_{1} \mathbf{R} \psi_{2}$ says that $\psi_{2}$ remains true until and including once $\psi_{1}$ becomes true; if $\psi_{1}$ never become true, $\psi_{2}$ must remain true forever. Regarding the "weak until" operator $\mathbf{W}$, the formula $\psi_{1} \mathbf{W} \psi_{2}$ is similar to $\psi_{1} \mathbf{U} \psi_{2}$ but the stop condition $\psi_{2}$ is not required to occur. In that case $\psi_{1}$ must remain true forever.

Distributivity laws:

$$
\begin{aligned}
\mathbf{X}\left(\psi_{1} \vee \psi_{2}\right) & \equiv\left(\mathbf{X} \psi_{1}\right) \vee\left(\mathbf{X} \psi_{2}\right) & \mathbf{X}\left(\psi_{1} \wedge \psi_{2}\right) & \equiv\left(\mathbf{X} \psi_{1}\right) \wedge\left(\mathbf{X} \psi_{2}\right) \\
\mathbf{X}\left(\psi_{1} \mathbf{U} \psi_{2}\right) & \equiv\left(\mathbf{X} \psi_{1}\right) \mathbf{U}\left(\mathbf{X} \psi_{2}\right) & \mathbf{X}\left(\psi_{1} \mathbf{R} \psi_{2}\right) & \equiv\left(\mathbf{X} \psi_{1}\right) \mathbf{R}\left(\mathbf{X} \psi_{2}\right) \\
\mathbf{F}\left(\psi_{1} \vee \psi_{2}\right) & \equiv\left(\mathbf{F} \psi_{1}\right) \vee\left(\mathbf{F} \psi_{2}\right) & \mathbf{G}\left(\psi_{1} \wedge \psi_{2}\right) & \equiv\left(\mathbf{G} \psi_{1}\right) \wedge\left(\mathbf{G} \psi_{2}\right) \\
\psi \mathbf{U}\left(\psi_{1} \vee \psi_{2}\right) & \equiv\left(\psi \mathbf{U} \psi_{1}\right) \vee\left(\psi \mathbf{U} \psi_{2}\right) & \left(\psi_{1} \wedge \psi_{2}\right) \mathbf{U} \psi & \equiv\left(\psi_{1} \mathbf{U} \psi\right) \wedge\left(\psi_{2} \mathbf{U} \psi\right)
\end{aligned}
$$

Negation propagation laws:

$$
\begin{aligned}
& \neg \mathbf{X} \psi_{1} \equiv \mathbf{X} \neg \psi_{1} \\
& \neg \mathbf{G} \psi_{1} \equiv \mathbf{F} \neg \psi_{1} \\
& \neg \mathbf{F} \psi_{1} \equiv \mathbf{G} \neg \psi_{1} \\
& \neg\left(\psi_{1} \mathbf{U} \psi_{2}\right) \equiv\left(\neg \psi_{1} \mathbf{R} \neg \psi_{2}\right) \\
& \neg\left(\psi_{1} \mathbf{R} \psi_{2}\right) \equiv\left(\neg \psi_{1} \mathbf{U} \neg \psi_{2}\right)
\end{aligned}
$$

Absorption laws:

$$
\begin{array}{rlrl}
\mathbf{F} \mathbf{F} \psi & \equiv \mathbf{F} \psi & \mathbf{G} \mathbf{G} \psi \equiv \mathbf{G} \psi \\
\mathbf{F G} \mathbf{F} \psi & \equiv \mathbf{G F} \psi & \mathbf{G F G} \psi & \equiv \mathbf{F} \mathbf{G} \psi
\end{array}
$$

Expansion laws:

$$
\psi_{1} \mathbf{U} \psi_{2} \equiv \psi_{2} \vee\left(\psi_{1} \wedge \mathbf{X}\left(\psi_{1} \mathbf{U} \psi_{2}\right)\right) \quad \neg\left(\psi_{1} \mathbf{U} \psi_{2}\right) \equiv \neg \psi_{2} \wedge\left(\neg \psi_{1} \vee \mathbf{X} \neg\left(\psi_{1} \mathbf{U} \psi_{2}\right)\right)
$$

For $\sim \in\{<, \leq,>, \geq\}$ define the operators $\approx$ and $[\sim]$ as follows:

$$
\begin{array}{rrrr}
\overline{<}=\geq & \overline{\leq}=> & \overline{>}=\leq & \bar{x}=< \\
{[<]=>} & {[\leq]=\geq} & {[>]=<} & {[\geq]=\leq}
\end{array}
$$

Some of the following equivalences cannot be used for "model checking" PCTL* (the left (P1) equivalence, to be specific) where actions are implicitly universally quantified. In the context of Markov Chains, which we implicitly have, there is no problem:

$$
\begin{aligned}
& \neg \mathbf{P}_{\sim z} \psi \equiv \mathbf{P}_{\bar{\sim}_{z}} \psi \\
& \mathbf{P}_{\geq 0} \psi \equiv \text { true } \\
& \mathbf{P}_{\sim z} \neg \psi \equiv \mathbf{P}_{[\sim] 1-z} \psi \\
& \mathbf{P}_{\leq 1} \psi \equiv \text { true } \\
& \mathbf{P}_{>1} \psi \equiv \text { false } \\
& \mathbf{P}_{<0} \psi \equiv \text { false } \\
& \mathbf{P}_{\geq u} \mathbf{P}_{\sim z} \psi \equiv \mathbf{P}_{\sim z} \psi \quad \text { if } u \neq 0 \\
& \mathbf{P}_{>u} \mathbf{P}_{\sim z} \psi \equiv \mathbf{P}_{\sim z} \psi \quad \text { if } u \neq 1 \\
& \mathbf{P}_{\leq u} \mathbf{P}_{\sim z} \psi \equiv \mathbf{P}_{\geq 1-u} \mathbf{P}_{\bar{\sim}} \psi \\
& \mathbf{P}_{<u} \mathbf{P}_{\sim z} \psi \equiv \mathbf{P}_{>1-u} \mathbf{P}_{\bar{\sim}_{z}} \psi
\end{aligned}
$$

Some notes on these equivalences. The left equivalence of $(\mathrm{P} 1)$ is trivial and the right equivalence uses the fact that $\operatorname{Pr}^{\mathcal{M}_{\pi}}\left\{s \cdots\left|\mathcal{M}_{\pi}, s \cdots\right|=\psi\right\}+\operatorname{Pr}^{\mathcal{M}_{\pi}}\left\{s \cdots\left|\mathcal{M}_{\pi}, s \cdots\right|=\neg \psi\right\}=1$. The equivalences (P2) and (P3) are again trivial. For the equivalences (P4) it helps to observe that the subformula $\mathbf{P}_{\sim z} \psi$ is both a state and a path formula. As a state formula it evaluates in a given context $\mathcal{M}, \pi, s$ to either true or false. Taken as a path formula of the outer $\mathbf{P}$ quantifier it hence stands for the set of either all runs from $s$ or the empty set, respectively. With this observation the equivalences (P4) follow easily from the semantics of the $\mathbf{P}$ operator. The equivalences (P5) are obtained by first applying the right equivalence in (P1) (from right to left) and then the left equivalence in (P1). 


\section{Example}

Consider the MDP in Figure 2 and the partially specified finite-memory policy $\pi_{\text {fin }}=(\{\mathrm{m}\}$, start, $\Delta, \cdot)$ with a single mode $\mathrm{m}$, making $\pi_{\mathrm{fin}}$ Markovian. The functions start and $\Delta$ hence always return $\mathrm{m}$, allowing us to abbreviate $\left\langle m, s_{i}\right\rangle$ as just $s_{i}$. Action $\beta$ leads non-deterministically to states $s_{2}$ and $s_{3}$, each with probability 0.5 . The actions $\alpha_{i}$ for $i \in\{1,2,3\}$ are self-loops with probability one (not shown). The label set of $s_{2}$ is $\{a\}$ in all other states it is empty.

The example is admittedly simple and is only from the PCTL subset of CTL*. But it suffices to show the main aspects of the calculus.

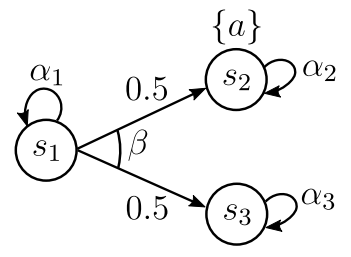

Figure 2: The transitions of the example MDP $\mathcal{M}$ depicted as a graph. The initial state is $s_{1}$. Action $\beta$ leads non-deterministically to states $s_{2}$ and $s_{3}$, each with probability 0.5 . The actions $\alpha_{i}$ for $i \in\{1,2,3\}$ are self-loops with probability one (not shown). The label set of $s_{2}$ is $\{a\}$ in all other states it is empty.

Let the state formula of interest be $\phi=\mathbf{P}_{\geq 0.3} \mathbf{F G} a$. We wish to obtain a $\Gamma_{\text {final }}$ such that any solution $\sigma$ synthesizes a suitable $\operatorname{act}_{\sigma}$, i.e., the policy $\pi_{\text {fin }}(\sigma)$ completed by $\sigma$ satisfies $\mathcal{M}_{\pi_{\text {fin }}(\sigma)}, s_{1} \mid=\phi$.

The BSCCs depend on whether act ${ }_{\sigma}\left(\mathrm{m}, s_{1}, \beta\right)>0$ holds, i.e., if $\beta$ can be executed at $s_{1}$. (This is why the calculus needs to make a corresponding guess, with its A-rule.) If not, then $s_{2}$ and $s_{3}$ are unreachable, and the self-loop at $s_{1}$ is the only BSCC, which does not satisfy $\mathbf{G} a$. If yes, then there are two BSCCs, the self-loop at $s_{2}$ and the self-loop at $s_{3}$, and the BSCC at $s_{2}$ satisfies $\mathbf{G} a$. By fairness of execution, with probability one some BSCC will be reached, and the BSCC at $s_{2}$ is reached with probability 0.5 , hence, if $\operatorname{act}_{\sigma}\left(\mathrm{m}, s_{1}, \beta\right)>0$. In other words, devising any policy that reaches $s_{2}$ will hence suffice to satisfy $\phi$. The expected result thus is just a constraint on $\sigma$ saying $\operatorname{act}_{\sigma}\left(\mathrm{m}, s_{1}, \beta\right)>0$ and the derivation will indeed show that by CHooseing a branch with $x_{s_{1}}^{\beta}>0$ in $\Gamma_{\text {final }}$.

Figures 3 to 7 summarize the derivation from the initial sequent $\sigma_{1}=x_{s_{1}}^{\{\phi\}} \doteq 1 \vdash s_{1}: \phi$. In these figures we write, for brevity, $\Gamma \vdash \psi, \Psi$ instead of $\Gamma \vdash\{\psi\} \uplus \Psi$. For better readability we write $\mathbf{G} \psi$ as a macro for $\neg \mathbf{F} \neg \psi$ and $\mathbf{F} \psi$ for true $\mathbf{U} \psi$. Please consider the captions in the figures for further explanations on notation.

Tree 1 in Figure 3 shows the derivation of Choose $\left(\operatorname{Tableau}\left(\sigma_{1}\right)\right)$. It has only one branch which ends in the leaf node $u_{\mathbb{1}}$. Because there are no BSCCs in tree (1), ForCE does not add constraints, and therefore $\Gamma_{\text {final }}=\Gamma_{u_{\Phi}}$ (the constraint system of $u_{\mathbb{1}}$ ). Notice that if the derivation had CHoosen the right branch at the top of tree $11, \Gamma_{\text {final }}$ would be unsatisfiable (as indicated in Figure 3). This case is uninteresting and we do not consider it further.

Figures 4-7 in combination show the derivation from initial sequent $\emptyset \vdash s_{1}: \mathbf{F G} a$ for computing $\Gamma_{2}$ in Figure 3. The dotted line in Figure 4 represents the contribution of tree (3) to 


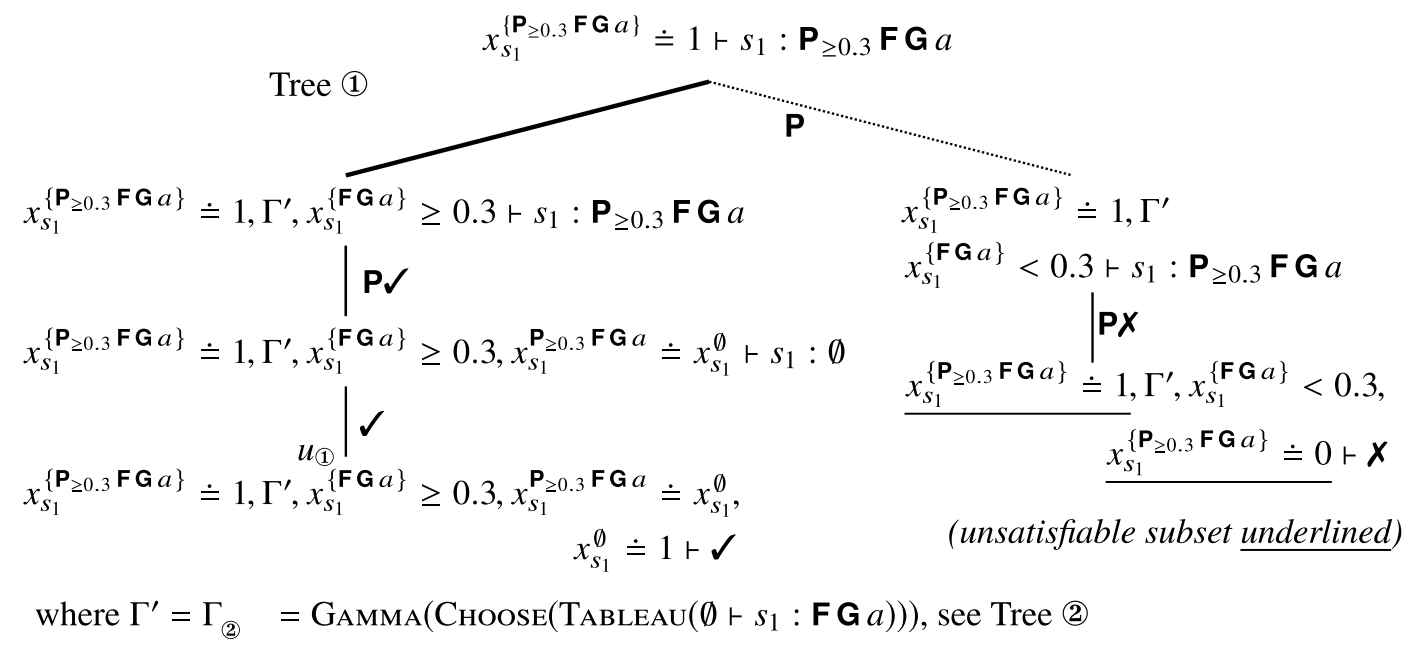

Figure 3: Start of derivation for synthesizing a policy for the MDP in Figure 2 for the formula $\mathbf{P}_{\geq 0.3} \mathbf{F G} a$. The links are annotated with the name of the inference rule applied. The CHoosen alternative in the $\mathbf{P}$ inference is the bold link, the dotted link is the non-CHoosen alternative. The leaf node of the left branch is called $u_{(\mathbb{1})}$.

tree (2) in terms of probabilities for the pivot $s_{1}: \mathbf{F G} a$ in the root of tree (2). More precisely, the constraint $\Gamma_{2}$ will enforce $x_{s_{1}}^{\{\mathrm{FG} a\}}=x_{s_{1}}^{\{\mathrm{XFG} a\}}$, where $x_{s_{1}}^{\{\mathrm{XFG} a\}}$ holds the probability of $s_{1}: \mathbf{X ~ F ~ G ~} a$ as per tree (3). This equality holds because all branches in tree (2) are $\boldsymbol{X}$-ed, therefore each contributing a value 0 to the sums in the union branches. (Figures 6 and 7 have more such examples.)

Figure 5 has the tree (3) with pivot $s_{1}: \mathbf{X F G} a$ at its root. Again the CHoosen alternatives are already highlighted, this time for the $\mathrm{A}$ inferences. (CHooseing the $x_{s_{1}}^{\alpha_{1}} \doteq 0$ branch would also lead to a policy, but obviously $\beta$ must be prescribed in $s_{1}$ for being able to reach $s_{2}$.)

It is instructive to see how tree (3) contributes to $\Gamma_{2}$ a constraint for $x_{S_{1}}^{\{\mathrm{XFG} a\}}$, i.e., the pivot at its root. For that, assume that the trees annotated as BSCC with (without) Yes-Loop all contribute a probability of one (zero, respectively) to $\Gamma_{2}$. (We spell this out in detail only in one case, cf. tree (4) in Figure 6.) Combining all constraints in the leaves of tree (3) then entails the following set of equalities (the comment $u_{1} \leftarrow u_{2}, u_{3}, u_{4}$ indicates the dependency of the left hand side of 


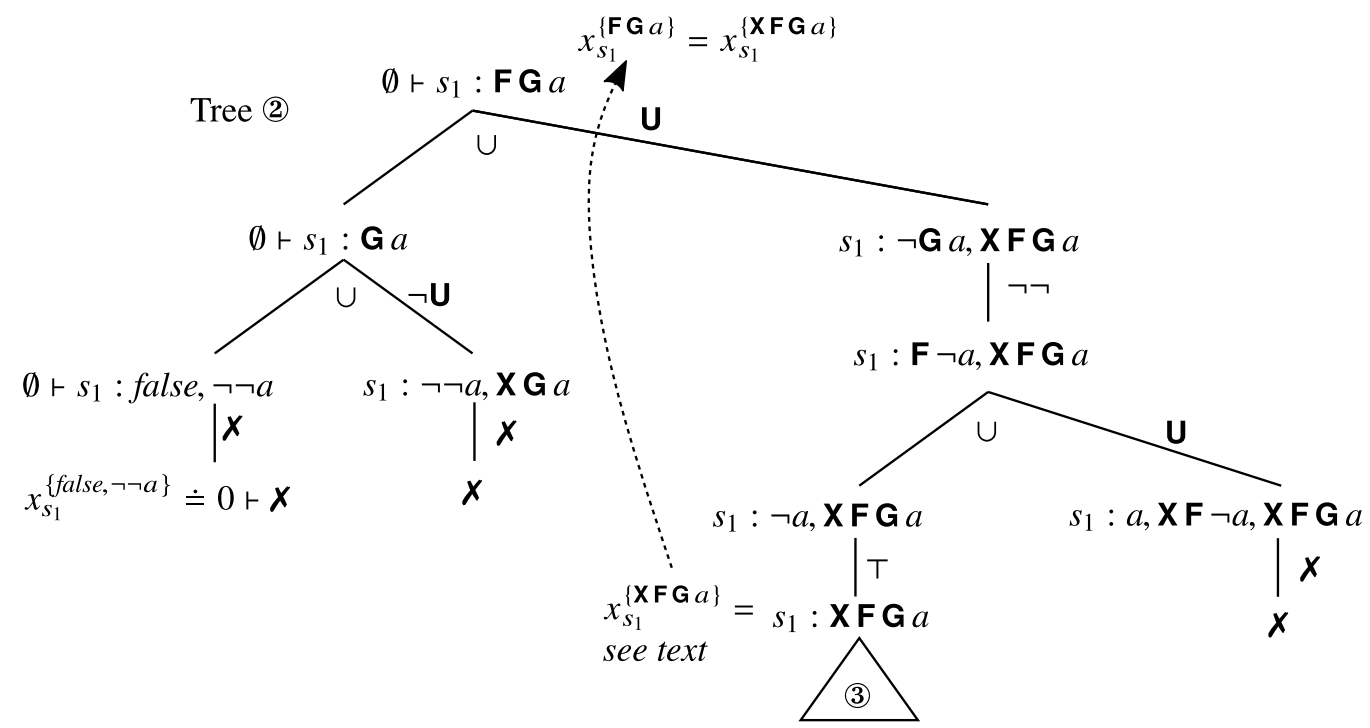

Figure 4: First part of a derivation from $\emptyset \vdash s_{1}: \mathbf{F G} a$ for computing $\Gamma_{(2)}$ in Figure 3. For space reasons we only write the right hand sides of sequences or only write interesting parts of the left hand sides. The dotted line represents a dependency of probability constraints. The derivation for tree (3) is in Figure 5.

its equality from the right hand side in terms of corresponding nodes):

$$
\begin{aligned}
& \left(x_{u_{1}}\right)_{s_{1}}^{\{\mathbf{X F G} a\}}=x_{s_{1}}^{\alpha_{1}} \cdot\left(x_{u_{2}}\right)_{s_{1}}^{\{\mathbf{F G} a\}}+x_{s_{1}}^{\beta} \cdot 0.5 \cdot\left(x_{u_{3}}\right)_{s_{2}}^{\{\mathbf{F G} a\}}+x_{s_{1}}^{\beta} \cdot 0.5 \cdot\left(x_{u_{4}}\right)_{s_{3}}^{\{\mathbf{F G} a\}} \\
& \left(x_{u_{2}}\right)_{s_{1}}^{\{\mathbf{F G} a\}}=\left(x_{u_{5}}\right)_{s_{1}}^{\{\mathbf{X F G} a\}} \quad\left(u_{2} \leftarrow u_{5}\right) \\
& \left(x_{u_{3}}\right)_{s_{2}}^{\{\mathbf{F} \mathbf{G} a\}}=1 \\
& \left(x_{u_{4}}\right)_{s_{3}}^{\{\mathbf{F} \mathbf{G} a\}}=0 \\
& \left(x_{u_{5}}\right)_{s_{1}}^{\{\mathbf{X F G} a\}}=x_{s_{1}}^{\alpha_{1}} \cdot\left(x_{u_{6}}\right)_{s_{1}}^{\{\mathbf{X F G} a\}}+x_{s_{1}}^{\beta} \cdot 0.5 \cdot\left(x_{u_{7}}\right)_{s_{2}}^{\{\mathrm{FG} a\}}+x_{s_{1}}^{\beta} \cdot 0.5 \cdot\left(x_{u_{8}}\right)_{s_{3}}^{\{\mathrm{FG} a\}} \\
& \left(x_{u_{6}}\right)_{s_{1}}^{\{\mathbf{X F G} a\}}=\left(x_{u_{9}}\right)_{s_{1}}^{\{\mathbf{X F G} a\}} \\
& \left(x_{u_{7}}\right)_{S_{2}}^{\{\mathbf{F} \mathbf{G} a\}}=1 \\
& \left(x_{u_{8}}\right)_{s_{3}}^{\{\mathbf{F G} a\}}=0 \\
& \left(x_{u_{9}}\right)_{s_{1}}^{\{\mathbf{X F G} a\}}=\left(x_{u_{1}}\right)_{s_{1}}^{\{\mathbf{X F G} a\}} \\
& \left(u_{5} \leftarrow u_{6}, u_{7}, u_{8}\right) \\
& \left(u_{6} \leftarrow u_{9}\right) \\
& \left(u_{9} \leftarrow u_{1}\right)
\end{aligned}
$$

Substituting in yields a simplified set of equalities:

$$
\begin{aligned}
& \left(x_{u_{1}}\right)_{s_{1}}^{\{\mathbf{X F G} a\}}=x_{s_{1}}^{\alpha_{1}} \cdot\left(x_{u_{5}}\right)_{s_{1}}^{\{\mathbf{X F G} a\}}+0.5 \cdot x_{s_{1}}^{\beta} \\
& \left(u_{1} \leftarrow u_{2}, u_{3}, u_{4}\right) \\
& \left(x_{u_{5}}\right)_{s_{1}}^{\{\mathbf{X F G} a\}}=x_{s_{1}}^{\alpha_{1}} \cdot\left(x_{u_{1}}\right)_{s_{1}}^{\{\mathbf{X F G} a\}}+0.5 \cdot x_{s_{1}}^{\beta} \\
& \left(u_{5} \leftarrow u_{6}, u_{7}, u_{8}\right)
\end{aligned}
$$




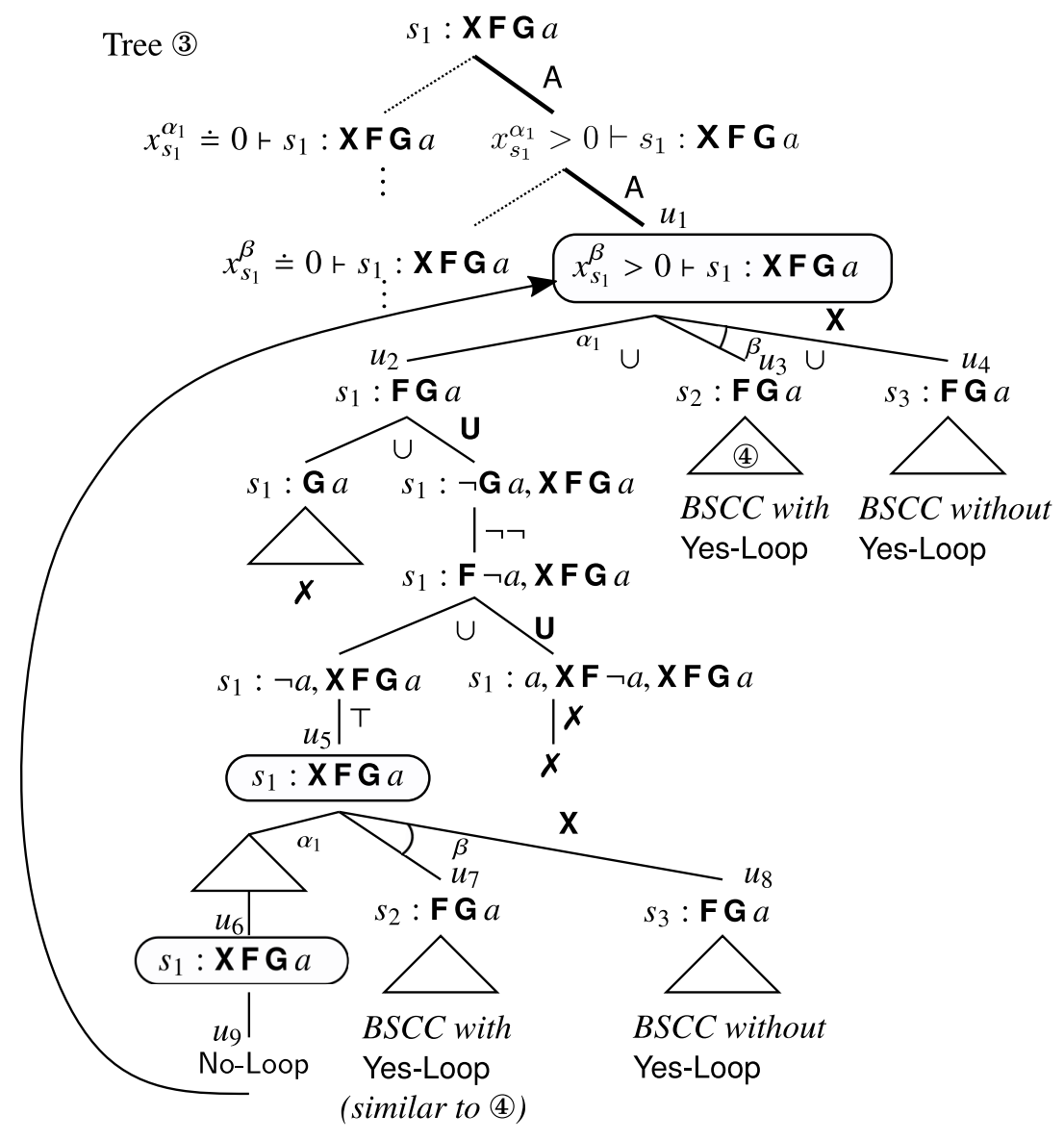

Figure 5: Sub-derivation with Tree (3) continuing Figure 4. Poised nodes are framed. The links in the $\mathbf{X}$ inference are annotated with actions. An triangle with a $\boldsymbol{X}$ is a tree all whose leaves are $\boldsymbol{X}$-ed. A triangle with links at the bottom is a tree with branches into the linked nodes and all whose leaves are $\boldsymbol{X}$-ed. A triangle labelled "BSCC with/without a Yes-Loop" is a tree that contains a BSCC with/without Yes-Loop and all non-loop leaves are $\boldsymbol{X}$-ed. Alternative choices for the A rule are only indicated as dots, as they are not relevant in this example. See text for propagation of constraints. 
Isolating $\left(x_{u_{1}}\right)_{s_{1}}^{\{\mathbf{X F G} a\}}$ on the left hand side:

$$
\left(x_{u_{1}}\right)_{s_{1}}^{\{\mathrm{XFG} a\}} \cdot\left(1-\left(x_{s_{1}}^{\alpha_{1}}\right)^{2}\right)=0.5 \cdot x_{s_{1}}^{\beta} \cdot\left(1+x_{s_{1}}^{\alpha_{1}}\right)
$$

Both $\alpha_{1}$ and $\beta$ are prescribed actions thanks to the two $\mathrm{A}$ inferences preceding the node $u_{1}$, resulting in $\left\{x_{s_{1}}^{\alpha_{1}}>0, x_{s_{1}}^{\beta}>0\right\} \subset \Gamma_{(2)}$. The $\mathbf{X}$ inference at $u_{1}$ adds the constraint $x_{s_{1}}^{\alpha_{1}}+x_{s_{1}}^{\beta} \doteq 1$ to $\Gamma_{(2)}$ (cf. $\gamma_{2}$ in the definition of the $\mathbf{X}$ rule). It follows $x_{s_{1}}^{\beta}=1-x_{s_{1}}^{\alpha_{1}}$. Substituting into the previous equality we get

$$
\left(x_{u_{1}}\right)_{s_{1}}^{\{\mathbf{X F G} a\}} \cdot\left(1-\left(x_{s_{1}}^{\alpha_{1}}\right)^{2}\right)=0.5 \cdot\left(1-x_{s_{1}}^{\alpha_{1}}\right) \cdot\left(1+x_{s_{1}}^{\alpha_{1}}\right)=0.5 \cdot\left(1-\left(x_{s_{1}}^{\alpha_{1}}\right)^{2}\right)
$$

Again from $x_{s_{1}}^{\alpha_{1}}+x_{s_{1}}^{\beta} \doteq 1$ and $x_{s_{1}}^{\beta}>0$ it follows $x_{s_{1}}^{\alpha_{1}}<1$ and thus $\left(1-\left(x_{s_{1}}^{\alpha_{1}}\right)^{2}\right)>0$. This allows us to divide both sides by this term and we simply get

$$
\left(x_{u_{1}}\right)_{s_{1}}^{\{\mathbf{X F G} a\}}=0.5
$$

Moreover, from the simplified constraints above, we have:

$$
\left(x_{u_{5}}\right)_{s_{1}}^{\{\mathbf{X F G} a\}}=x_{s_{1}}^{\alpha_{1}} \cdot\left(x_{u_{1}}\right)_{s_{1}}^{\{\mathbf{X F G} a\}}+0.5 \cdot x_{s_{1}}^{\beta}=0.5 \cdot\left(x_{s_{1}}^{\alpha_{1}}+x_{s_{1}}^{\beta}\right)=0.5
$$

From tree (2) we get $\left(x_{u_{2}}\right)_{s_{1}}^{\{\mathbf{F G} a\}}=\left(x_{u_{5}}\right)_{s_{1}}^{\{\mathbf{X F G} a\}}$. Substituting into $x_{s_{1}}^{\{\mathbf{F G} a\}} \geq 0.3$ from tree (1) we get a tautology.

Altogether, the only non-trivial constraints in $\Gamma_{\text {final }}$ are those introduced by the various $A$ inferences for constraining probabilities of actions. For the concretely CHOosen alternatives in the example, this means that any solution that satisfies $x_{s_{1}}^{\alpha_{1}}>0$ and $x_{s_{1}}^{\beta}>0$ provides a policy. Notice that only $x_{s_{1}}^{\beta}>0$ is essential, and CHooseing the alternative $x_{s_{1}}^{\alpha_{1}} \doteq 0$, which entails $x_{s_{1}}^{\beta}=1$, will do as well.

At this point it only remains to go through the derivations for tree (4) and (5). With the explanations so far this should be straightforward.

Figure 6 has the tree (4) for the pivot $s_{2}: \mathbf{F ~ G} a$. From the MDP in Figure 2 we expect it has probability one. It is formally computed by adding the probabilities of $s_{2}: \mathbf{G} a$, which is one, and of $s_{2}: \neg \mathbf{G} a, \mathbf{X} \mathbf{F} \mathbf{G} a$, which is zero. The latter requires identification of a BSCC without yes-loops, as depicted, which, hence, Forcees zero.

Similarly, Figure 7 has the tree (5) for the pivot $s_{1} s_{2}: \mathbf{G} a$. It is an example for a BSCC with a yes-loop. Notice that the poised pivot that forms the BSCC has no $\mathbf{X}$-eventualities at all. 


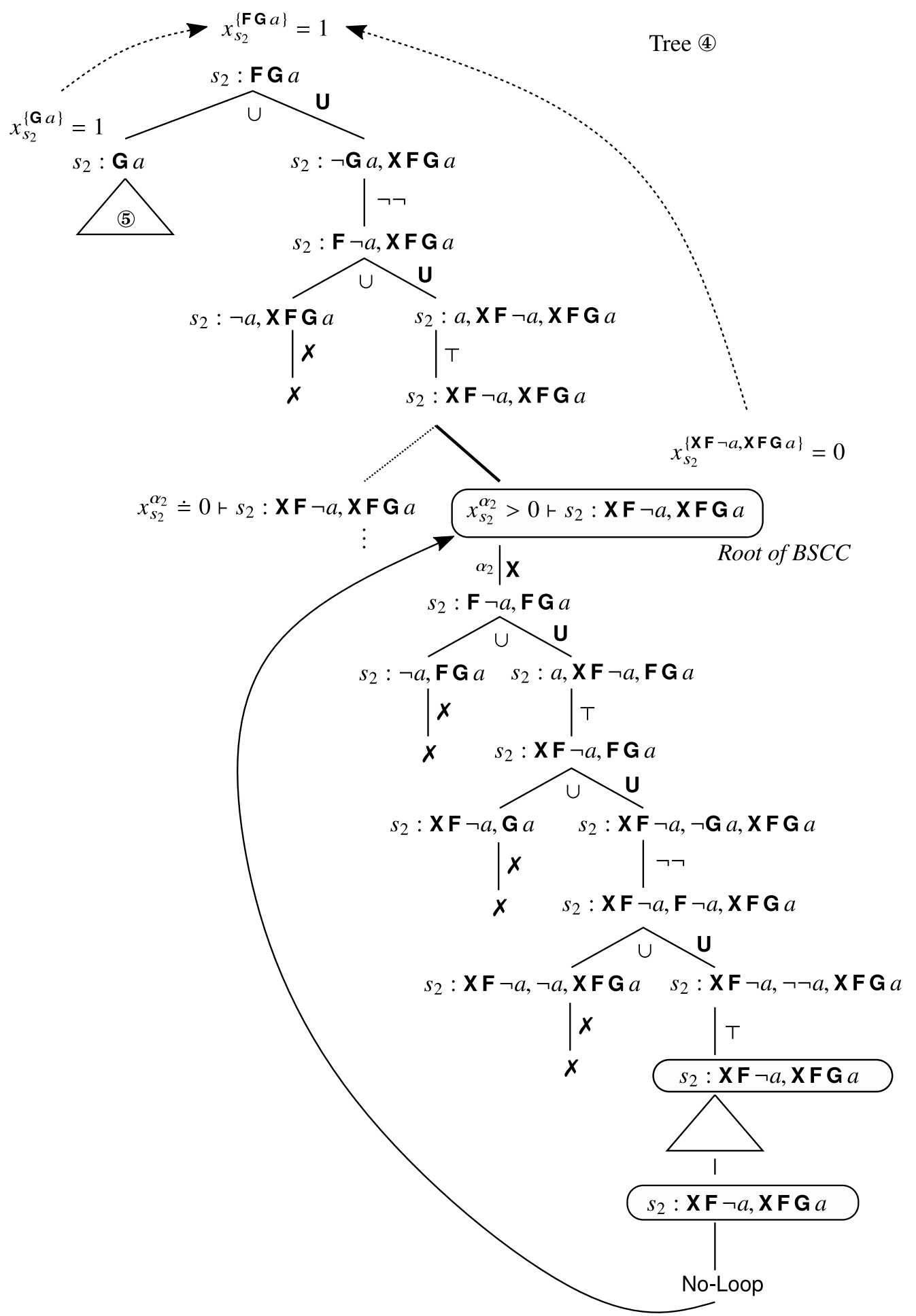

Figure 6: Sub-derivation with Tree (4) continuing Figure 5. 


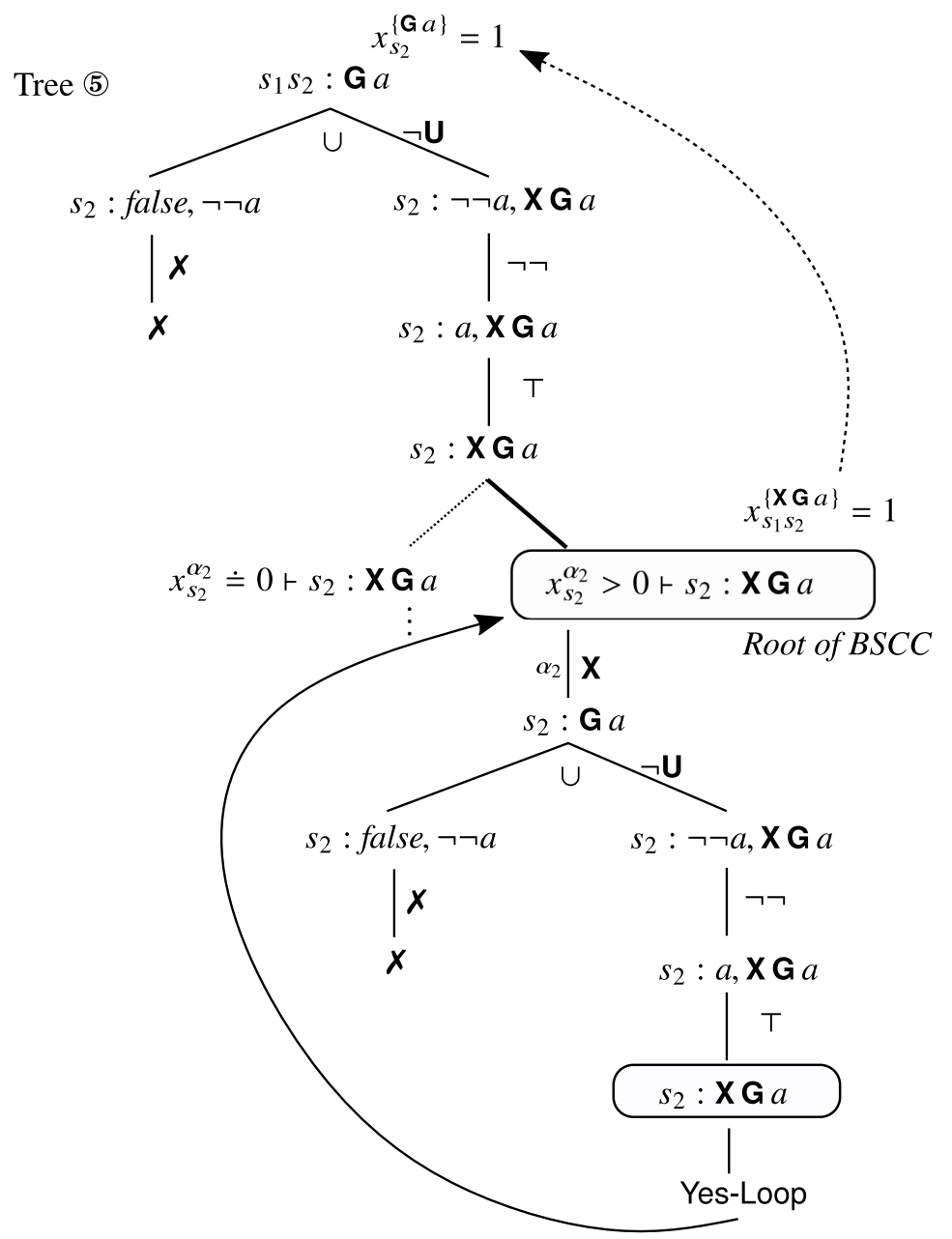

Figure 7: Sub-derivation with Tree (5) continuing Figure 6. 


\section{Proofs}

Lemma 7.1 Let $b$ be a branch in a tree with poised nodes $u$ and $w$ with $u<w$. If $\Psi_{u}=\Psi_{w}$ then for every poised node $v$ with $u<v<w$ it holds $\Psi_{v} \supseteq \Psi_{u}$.

Proof. Suppose $\Psi_{u}=\Psi_{w}$ and a poised node $v$ with $u<v<w$. Let $x$ be the $\mathbf{X}$-successor node of $u$ on $b$. The set $\Psi_{u}$ is of the form $\left\{\mathbf{X} \psi_{1}, \ldots, \mathbf{X} \psi_{n}\right\}$ and, hence, $\Psi_{x}=\left\{\psi_{1}, \ldots, \psi_{n}\right\}$.

Now consider the available inference rules. It is straightforward to check that every inference rule except $\mathbf{U}$ and $\neg \boldsymbol{U}$ either leaves the pivot $\langle m, s\rangle: \Psi$ untouched or replaces some $\psi \in \Psi$ by zero or more strictly simpler formulas. With " $\psi_{1}$ is simpler than $\psi_{2}$ " we mean that $\psi_{1}$ has strictly less symbols than $\psi_{2}$ or else the number of symbols are the same but a negation sign in $\psi_{1}$ has been pushed inwards to get $\psi_{2}$ (this is needed for the $\neg \mathbf{X}$ rule).

The $\mathbf{U}$ and $\neg \mathbf{U}$ rules also add simpler subformulas to the conclusion, together with an $\mathbf{X}$ operator in front of the $\mathbf{U}$ formula or negated $\mathbf{U}$ formula it was applied to.

The $\mathbf{U}$ and $\neg \mathbf{U}$ rules are the only ones that can restore the $\mathbf{X}$-operator applications in $\Psi_{w}=$ $\left\{\mathbf{X} \psi_{1}, \ldots, \mathbf{X} \psi_{n}\right\}$. In other words, the ancestor nodes of $w$ must collectively contain the formulas $\psi_{1}, \ldots, \psi_{n}$ in their formula sets and, furthermore, every $\psi_{i}$ is an $\mathbf{U}$-formula or a negated $\mathbf{U}$-formula.

Because it is impossible to derive a $\mathbf{U}$-formula or a negated $\mathbf{U}$-formula from simpler formulas (there is just no inference rule capable of that) this means they must have been present from the beginning. No $\psi_{i} \in \Psi_{x}$ can have been replaced by simpler formulas from $x$ down to $w$. Furthermore, every $\psi_{i}$ must have been subject to a $\mathbf{U}$ and $\neg \mathbf{U}$ inference above the node $v$, otherwise $v$ is not poised. It follows $\Psi_{v} \supseteq \Psi_{u}$.

Theorem 3.1 (Soundness) Let $\mathcal{M}=\left(S, s_{\mathrm{init}}, A, P, L\right)$ be an $M D P, \pi_{\mathrm{fin}}=(M, \operatorname{start}, \Delta, \cdot)$ be a partially specified finite-memory policy with act unspecified, and $\phi$ a state formula. Suppose there is a program $\Gamma_{\text {final }}:=\operatorname{Gamma}\left(\operatorname{CHOOSE}\left(\operatorname{TableaU}\left(\left\{x_{\left\langle\text {start }\left(s_{\text {init }}\right), s_{\text {init }}\right\rangle} \doteq 1\right\} \vdash\left\langle\operatorname{start}\left(s_{\text {init }}\right), s_{\text {init }}\right\rangle\right.\right.\right.$ : $\{\phi\}))$ ) such that $\Gamma_{\text {final }}$ is satisfiable. Let $\sigma$ be any solution of $\Gamma_{\text {final }}$ and $\pi_{\mathrm{fin}}(\sigma)$ be the policy completed by $\sigma$. Then it holds $\mathcal{M}, \pi_{\mathrm{fin}}(\sigma) \mid=\phi$.

Proof. (Sketch) Let $\Gamma_{\text {final }}, \sigma$ and $\pi_{\text {fin }}(\sigma)$ as stated. We have to show $\mathcal{M}, \pi_{\text {fin }}(\sigma) \mid=\phi$, equivalently $\mathcal{M}_{\pi_{\text {fin }}(\sigma)},\left\langle\operatorname{start}\left(s_{\text {init }}\right), s_{\text {init }}\right\rangle \mid=\phi$.

We need to look at the collection of sub-derivations from the initial sequent. Let $\mathcal{T}_{0}, \mathcal{T}_{1}, \ldots, \mathcal{T}_{n}$ be a sequence of trees such that

$$
\begin{array}{ll}
\mathcal{T}_{0}=\operatorname{Choose}\left(\operatorname{TableaU}\left(\left\{x_{\left\langle\operatorname{start}\left(s_{\text {init }}\right), s_{\text {init }}\right\rangle} \doteq 1\right\} \vdash\left\langle\operatorname{start}\left(s_{\text {init }}\right), s_{\text {init }}\right\rangle:\{\phi\}\right)\right) \\
\mathcal{T}_{j}=\operatorname{Choose}\left(\operatorname{TableaU}\left(\emptyset \vdash\left\langle m_{j}, s_{j}\right\rangle:\left\{\psi_{j}\right\}\right)\right) & \text { for } j=1 \ldots n, \text { where } \mathcal{T}_{j} \text { comes } \\
& \text { from some } \mathbf{P} \text { inference in the } \\
& \text { derivation }
\end{array}
$$

For $i=0 \ldots n$ we call $\mathcal{T}_{i}$ a tree from $\Gamma_{i} \vdash\left\langle m_{i}, s_{i}\right\rangle:\left\{\psi_{i}\right\}$.

The sequence is meant to be minimal and closed under $\mathbf{P}$ inferences in subderivations. Formally, it is constructed inductively: starting from $\mathcal{T}_{0}$ one takes the $\mathbf{P}$ inferences in $\mathcal{T}_{0}$ and appends the trees for these $\mathbf{P}$ inferences. Then proceed to the next tree in the sequence, do the same, and so on, until all sub-derivations have been processed. 
We can accompany the sequence of trees with a sequence $\Gamma_{0}, \Gamma_{1}, \ldots, \Gamma_{n}$, where $\Gamma_{i}=$ $\operatorname{Gamma}\left(\mathcal{T}_{i}\right)$, for $i=0 \ldots n$. Together they represent a derivation from $\left\{x_{\left\langle\operatorname{start}\left(s_{\text {init }}\right), s_{\text {init }}\right\rangle}^{\{\phi 1\}}\right.$ $\left\langle\operatorname{start}\left(s_{\text {init }}\right), s_{\text {init }}\right\rangle:\{\phi\}$ where $\Gamma_{\text {final }}=\Gamma^{0}$.

To prove the theorem, we prove something more general. For all $i=0 \ldots n$, if $\Gamma \vdash\langle m, s\rangle: \Psi$ is the label of a node in $\mathcal{T}_{i}$ then:

(i) If $\Psi$ is a set of state formulas then $v \in\{0,1\}$ where $v=\sigma\left(x_{\langle m, s\rangle}^{\Psi}\right)$. Moreover, $v=1$ iff $M_{\pi_{\text {fin }}(\sigma)},\langle m, s\rangle \mid \wedge \Psi$. (And hence $v=0$ iff $M_{\pi_{\text {fin }}(\sigma)},\langle m, s\rangle \vDash \wedge \Psi$.)

(ii) If $\Psi$ contains at least one proper path formula then

$$
\sigma\left(x_{\langle m, s\rangle}^{\Psi}\right)=\operatorname{Pr}^{\mathcal{M}_{\pi_{\text {fin }}(\sigma)}}\left(\left\{r \in \text { Runs }^{\mathcal{M}_{\pi_{\text {fin }}(\sigma)}}(\langle m, s\rangle) \mid \mathcal{M}_{\pi_{\text {fin }}(\sigma)}, r \models \bigwedge \Psi\right\}\right)
$$

Notice that cases (i) and (ii) are exclusive and exhaustive. If $\Psi=\emptyset$ then case (i) applies.

For the proof, fix some $i \in\{0 \ldots n\}$ and let $\Gamma \vdash\langle m, s\rangle: \Psi$ be the label of a node in $\mathcal{T}_{i}$.

Proof of $(i)$. Suppose $\Psi$ is a set of state formulas. We prove the conclusion by induction on the structure of $\Psi$.

If $\Psi=\emptyset$ then with the $\checkmark$ rule this is trivial. If there is a classical $\phi \in \Psi$ then we have two cases: if $L(s) \mid=\phi$ (or equivalently: $\mathcal{M}_{\pi_{\text {fin }}(\sigma)},\langle m, s\rangle \mid=\phi$ ) then we get a successor node with a $T$ inference. The result follows by induction together with the constraint $\gamma_{\text {one }}$ of the T rule.

If $L(s) \notin \phi$ then with a $\boldsymbol{X}$ inference we get a constraint $x_{\langle m, s\rangle}^{\Psi} \doteq 0$ in $\Gamma_{\text {final }}$, which trivially gets the result. In particular it follows $M_{\pi_{\text {fin }}(\sigma)},\langle m, s\rangle \mid \vDash \wedge \Psi$.

Hence suppose now every $\phi \in \Psi$ is non-classical. Choose one such $\phi$. If one of the $\neg \neg$, $\neg \mathbf{P}, \mathbf{P} \neg, \wedge, \neg \wedge, \mathbf{P} 1, \mathbf{P} 2$ or $\mathbf{P} 3$ inference rules is applicable, we get in each of the (one or two) conclusions a set of state formulas. This is easy to verify by inspecting the inference rules. In each case the result follows by induction together with the new constraints introduced by the inference rules. We spell this out only in the most interesting case, the $\neg \wedge$ rule.

For the $\neg \wedge$ rule, the formula set $\Psi$ is of the form $\left\{\neg\left(\psi_{1} \wedge \psi_{2}\right)\right\} \cup \Psi^{\prime}$ and $\phi=\neg\left(\psi_{1} \wedge \psi_{2}\right)$. For the two conclusions we get results by induction. Notice that the left conclusion has $\neg \psi_{1}$ in its formula set $\left\{\neg \psi_{1}\right\} \cup \Psi^{\prime}$, whereas the right conclusion has the complement $\psi_{1}$ in its formula set $\left\{\psi_{1}, \neg \psi_{2}\right\} \cup \Psi^{\prime}$. This entails for the induction results that not both $M_{\pi_{\text {fin }}(\sigma)},\langle m, s\rangle \mid=\left\{\neg \psi_{1}\right\} \cup \Psi^{\prime}$ and $M_{\pi_{\text {fin }}(\sigma)},\langle m, s\rangle \mid=\left\{\psi_{1}, \neg \psi_{2}\right\} \cup \Psi^{\prime}$ can hold. In terms of corresponding variables this means $v_{l}, v_{r} \in\{0,1\}$ but $\left\{v_{l}, v_{r}\right\} \neq\{1\}$ where $v_{l}=\sigma\left(x_{\langle m, s\rangle}^{\left\{\neg \psi_{1}\right\} \cup \Psi^{\prime}}\right)$ and $v_{r}=\sigma\left(x_{\langle m, s\rangle}^{\left\{\psi_{1}, \neg \psi_{2}\right\} \cup \Psi^{\prime}}\right)$. Finally, with the definition of the constraint $\gamma$ in the definition of the $\neg \wedge$ rule the result follows easily.

The only missing case is when $\phi$ is of the form $\mathbf{P}_{\sim z} \psi$. By closure under the inference rules with higher priority (those mentioned above) we know that $\psi$ is a proper path formula.

If the $\mathbf{P}$ rule is applicable then the left conclusion adds the constraint $\gamma_{\text {left }}=x_{\langle m, s\rangle}^{\{\psi\}} \sim z$ and the right conclusion adds the constraint $\gamma_{\text {right }}=x_{\langle m, s\rangle}^{\{\psi\}} \approx z$. As the $\mathbf{P}$ is a don't know rule the tree $\mathcal{T}_{k}$ has CHoosen one of them.

In any case, the $\mathbf{P}$ rule invokes a tableau derivation which gives us some tree $\mathcal{T}_{m}$ from $\emptyset \vdash\langle m, s\rangle:\{\psi\}$ in the above sequence of trees. From property (ii) we get

$$
\sigma\left(x_{\langle m, s\rangle}^{\Psi}\right)=\operatorname{Pr}^{\mathcal{M}_{\pi_{\text {fin }}(\sigma)}}\left(\left\{r \in \text { Runs }^{\mathcal{M}_{\pi_{\text {fin }}(\sigma)}}(\langle m, s\rangle)\left|\mathcal{M}_{\pi_{\text {fin }}(\sigma)}, r\right|=\psi\right\}\right)
$$


In the left case, with $\left(x_{\langle m, s\rangle}^{\{\psi\}} \sim z\right) \in \Gamma_{\text {final }}$ and the semantics of the $\mathbf{P}$ operator it follows $\mathcal{M}_{\pi_{\text {fin }}(\sigma)},\langle m, s\rangle \mid=\mathbf{P}_{\sim z} \psi$. (Recall that $\Gamma_{\text {final }}$ is satisfiable.) With a, not necessarily immediately, following $\mathbf{P} T$ inference the situation is the same as above, where we had the classical formula $\phi$ such that $\mathcal{M}_{\pi_{\text {fin }}(\sigma)},\langle m, s\rangle \mid=\phi$ and a $T$ inference. As above, the result follows by induction.

In the right case, with $\left(x_{\langle m, s\rangle}^{\{\psi\}} \approx z\right) \in \Gamma_{\text {final }}$ and the semantics of the $\mathbf{P}$ operator it follows $\mathcal{M}_{\pi_{\text {fin }}(\sigma)},\langle m, s\rangle \mid \vDash \mathbf{P}_{\sim z} \psi$. With a (not necessarily immediately) following $\mathbf{P} \boldsymbol{X}$ the result follows trivially.

If the $\mathbf{P}$ rule is not applicable then $\Gamma$ contains $\gamma_{\text {left }}$ or $\gamma_{\text {right }}$ already, but the same argumentation as for the left/right case applies.

This concludes the proof of property (i).

Proof of (ii). The proof is similar to the proof of (i) except for BSCCs, which requires special consideration.

Let $u$ be the node in $\mathcal{T}_{i}$ labelled with $\Gamma \vdash\langle m, s\rangle: \Psi$, the sequent we are looking at.

What we do is induction on the structure of $\Psi$, much like in case (i). We are adding up probabilities from the children of a union-branching to the parent node, in terms of corresponding variables. That this is correct is clear from inspecting the design of the inference rules with respect to the PCTL* semantics. Verifying the $\mathbf{X}$ rule may require a little closer look, though, but is not too difficult. An important detail is that the branching out into $\mathbf{X}$-successor nodes happens according to prescribed actions, by preference of the $A$ rule over the $\mathbf{X}$ rule. If, say, $\alpha \in \operatorname{Prescribed}(\Gamma,\langle m, s\rangle)$ is a prescribed action, then $\Gamma$ (and hence $\Gamma_{\text {final }}$ ) contains a variable $x_{\langle m, s\rangle}^{\alpha}$. It follows that the policy $\pi_{\text {fin }}(\sigma)$ will accordingly have act $\sigma(m, s, \alpha)=\sigma\left(x_{\langle m, s\rangle}^{\alpha}\right)$ rather than an arbitrary choice for making it complete.

If $u$ is also the root of a BSCC in $\mathcal{T}_{i}$ then Forceing adds $x_{\langle m, s\rangle}^{\Psi} \doteq 1$ or $x_{\langle m, s\rangle}^{\Psi} \doteq 0$ to $\operatorname{Gamma}\left(\mathcal{T}_{i}\right)$ and hence also to $\Gamma_{\text {final }}$. This entails $\sigma\left(x_{\langle m, s\rangle}^{\Psi}\right)=\chi$ where $\chi$ or $\chi=0$, respectively. For proving (ii) this means we need to show

$$
\operatorname{Pr}^{\mathcal{M}_{\pi_{\text {fin }}(\sigma)}}\left(\left\{r \in \text { Runs }^{\mathcal{M}_{\pi_{\text {fin }}(\sigma)}}(\langle m, s\rangle)\left|\mathcal{M}_{\pi_{\text {fin }}(\sigma)}, r\right|=\bigwedge \Psi\right\}\right)=\chi
$$

Let us start by some considerations about runs in relation to the BSCC rooted at $u$. Consider any run $r$ from $\langle m, s\rangle$. We can trace $r$ 's state transitions in Subtree $(u)$ as follows: at a poised inner node (such as $u$ ) select the proper $\mathbf{X}$-successor node, say, $v$ as given by the state transition in $r$ we are currently looking at (initially the first one, from $u$ ). Now consider Subtree(v). It cannot be a 0-deadend by definition of ambiguity. In other words, some of its branches may lead to a $\boldsymbol{X}$-ed leaf without encountering a poised inner node, but not all of them (and no branch has a $\checkmark$-ed leaf either). For any branch of the latter kind we can find the first poised node as we go down. Let $v_{1}, \ldots, v_{n}$ be all these poised fringe nodes, for some $n \geq 1$. We can view Subtree( $v$ ) as an analysis of the Boolean structure until reaching a fringe node. The path component does not advance during that, and so the fringe nodes each have the same path component as $v$. This is why we can take any $v_{i}$ for continuing the tracing (with $p_{v_{i}}=p_{v}$ ). Should $v_{i}$ be a leaf node we need to follow its backlink to the inner, indistinguishable node first.

This way we can follow $r$ stepwise in the BSCC rooted at $u$.

Let us consider the case $\chi=1$ in (1) above. That is, we have a Yes-Loop.

Should we have the freedom to construct $r$ as we wish, we could do it in such a way that it always passes through the (or one of the) Yes-Loop leaves. This would give us what is sometimes 
called a "lasso": from $u$ go down the proper branch and find that inner node, say $w(u=w$ is possible), that the Yes-Loop leaf backjumps to. Then there is an initial segment from $u$ to $w$ followed by circles leading back to $u$. The run $r$ would just follows this "path" ad infinitum.

This situation is identified by Mark Reynolds for proving his tableau algorithm [17] correct. (The long version [18] has proofs.) Let us call his tableau "MR tableaux". An important difference is that [18] is concerned with LTL satisfiability, not model checking. (A minor difference is that we have additionally the $\mathbf{P}$ operator, which however behaves much like a propositional symbol by the recursive call to the algorithm, plus induction.) For the soundness proof he assumes as given an LTL formula and an MR tableau that has (in our words) a Yes-Loop leaf. From the Yes-Loop he constructs a lasso run as described above, derives states and a labelling function from it, and shows that this run satisfies the given LTL formula. He actually has a more general construction which annotates the states in the runs with poised formula sets and keeps track of the expansions by the $\mathbf{X}$ rule and all other subsequent inferences (which are similar to ours). See the "truth lemma", Lemma 3 in [18].

We wish to re-use the correctness results for the MR tableaux. The main difficulty is that, of course, we are not free to construct the run, instead it is given. More precisely, the given run $r$ might not only follow the lasso, it may include other segments leading to No-Loops as well. What we do know, however, is that for fairness reasons every leaf must be visited infinitely often (recall we are dealing with a BSCC). This means that the loop in the lasso must be executed infinitely often along $r$, but not necessarily consecutively.

Because of the just said the truth lemma is not immediately applicable to our run $r$. But we can think of the run $r$ as executing a modified Yes-Loop loop infinitely often, with execution segments detouring into No-Loops spliced in. By inspecting the proof of the truth lemma it becomes clear that this splicing-in does not hurt as long as some essential formulas are preserved between subsequent poised nodes. These formulas are used to establish that $\mathbf{U}$-formulas or negated $\mathbf{U}$-formulas, stemming from an $\mathbf{X}$ inference to a poised node, are satisfied by the run in the MR tableau. See Lemmas 2 and 3 in [18]. That these formulas are indeed preserved by our runs as well then follows from Lemma 7.1 above: if there are additional poised nodes spliced into the Yes-Loops, they are equal to or supersets of the original poised nodes and, therefore, harmless. In other words, it can be shown that our run $r$ satisfies $\Psi$.

Finally as our run $r$ is chosen arbitrarily, every run from last $(p)$ satisfies $\Psi$. As the probability of the set of all runs is one, we have shown (1) for the case $\chi=1$.

It remains to consider the case $\chi=0$. That is, we have no Yes-Loop in the BSCC rooted at $u$. Again we refer to the proofs in [18]. This time it is actually easier. The completeness proof in [18] (Lemma 5) needs to analyze an arbitrary given run and trace it down the branches in the MR tableau, much like our run $r$ is allowed to walk down any branch and return to an inner node from its leaves (which are all No-Loop). So this situation is a better match.

The main argument, in brief, is that there must be an $\mathbf{X}$-eventuality in $\Psi$ that remains unsatisfied along $r$, making $\Psi$ unsatisfied by $r$, if there is no Yes-Loop. For this it is shown that the loop-check does not prematurely cut branches. In brief, when a leaf is a No-Loop the branch leading to it repeatedly failed to satisfy an $\mathbf{X}$-eventuality and it would not help to make this same mistake twice.

Finally as our run $r$ is chosen arbitrarily, no run from $\langle m, s\rangle$ satisfies $\Psi$. As the probability 
of the empty set of runs is zero, we have shown (1) for the case $\chi=0$.

This concludes the proof of property (ii).

Finally it is easy to prove the conclusion $\mathcal{M}, \pi_{\mathrm{fin}}(\sigma) \mid=\phi$ of the theorem, which expands to the equivalent statement $\mathcal{M}_{\pi_{\text {fin }}(\sigma)},\left\langle\operatorname{start}\left(s_{\text {init }}\right), s_{\text {init }}\right\rangle \vDash \phi$. Because $\phi$ is a state formula we can apply property (i) to the tree $\mathcal{T}_{0}$ from $\left\{x_{\left\langle\text {start }\left(s_{\text {init }}\right), s_{\text {init }}\right\rangle} \doteq 1\right\} \vdash\left\langle\operatorname{start}\left(s_{\text {init }}\right), s_{\text {init }}\right\rangle:\{\phi\}$ and conclude $v \in\{0,1\}$ where $v=\sigma\left(x_{\left\langle\text {start }\left(s_{\text {init }}\right), s_{\text {init }}\right\rangle}\right)$. With $x_{\left\langle\text {start }\left(s_{\text {init }}\right), s_{\text {init }}\right\rangle}^{\left\{s_{1}\right.} \doteq 1 \in \Gamma_{\text {final }}$ trivially $v=1$. By property (i) again $\mathcal{M}_{\pi_{\text {fin }}(\sigma)},\left\langle\operatorname{start}\left(s_{\text {init }}\right), s_{\text {init }}\right\rangle \models \phi$.

Theorem 3.2 (Completeness) Let $\mathcal{M}=\left(S, s_{\text {init }}, A, P, L\right)$ be an $M D P, \pi_{\mathrm{fin}}=(M$, start, $\Delta$, act $) a$ finite-memory policy, and $\phi$ a state formula. Suppose $\mathcal{M}, \pi_{\mathrm{fin}}=\phi$. Then there is a satisfiable $\operatorname{program} \Gamma_{\text {final }}:=\operatorname{Gamma}\left(\operatorname{Choose}\left(\operatorname{TableaU}\left(\left\{x_{\left\langle\operatorname{start}\left(s_{\text {init }}\right), s_{\text {init }}\right\rangle} \doteq 1\right\} \vdash\left\langle\operatorname{start}\left(s_{\text {init }}\right), s_{\text {init }}\right\rangle:\{\phi\}\right)\right)\right)$ and a solution $\sigma$ of $\Gamma_{\text {final }}$ such that $\operatorname{act}_{\sigma}(m, s, \alpha)=\operatorname{act}(m, s, \alpha)$ for every pair $\langle m, s\rangle$ in the policy domain of $\Gamma_{\text {final }}$. Moreover $\mathcal{M}, \pi_{\text {fin }}(\sigma) \models \phi$.

Proof. (Sketch.) Let $\phi$ be as stated and suppose there is a finite-memory policy $\pi_{\text {fin }}$ such that $\mathcal{M}, \pi_{\text {fin }} \mid=\phi$. In other words $\mathcal{M}_{\pi_{\text {fin }}},\left\langle\operatorname{start}\left(s_{\text {init }}\right), s_{\text {init }}\right\rangle \mid=\phi$.

A preliminary: a binding is a pair $(x, v)$, usually written as $x \mapsto v$, where $x$ is a variable and $v \in[0,1]$. A substitution is a finite set of bindings. The set $\operatorname{dom}(\sigma)=\{x \mid(x \mapsto v) \in$ $\sigma$ for some $v\}$ is called the domain of $\sigma$. We use substitutions to construct solutions of programs.

The proof plan is to construct TABLEAU $\left(\left\{x_{\left\langle\text {start }\left(s_{\text {init }}\right), s_{\text {init }}\right\rangle} \doteq 1\right\} \vdash\left\langle\operatorname{start}\left(s_{\text {init }}\right), s_{\text {init }}\right\rangle:\{\phi\}\right)$ in a similar fashion as one would do in the soundness proof for classical tableau, by analysing $\phi$ syntactically and going down branches. It is a bit trickier in the completeness case, though. We have to CHOose along the way as needed for complying with the prescribed actions in the given policy $\pi_{\text {fin }}$ and the $\mathbf{P}$-formulas. We also have to show that the Gamma operations results in a satisfiable $\Gamma_{\text {final }}$. The rest of the proof spells this out in more detail.

We need something general to keep the induction going, as follows.

As in the soundness proof we work with a sequence of trees $\mathcal{T}_{0}, \mathcal{T}_{1}, \ldots, \mathcal{T}_{n}$ such that

$$
\begin{array}{ll}
\mathcal{T}_{0}=\operatorname{Choose}\left(\operatorname{Tableau}\left(\left\{x_{\left\langle\operatorname{start}\left(s_{\text {init }}\right), s_{\text {init }}\right\rangle}^{\{\phi\}} \doteq 1\right\} \vdash\left\langle\operatorname{start}\left(s_{\text {init }}\right), s_{\text {init }}\right\rangle:\{\phi\}\right)\right) \\
\mathcal{T}_{j}=\operatorname{Choose}\left(\operatorname{Tableau}\left(\emptyset \vdash\left\langle m_{j}, s_{j}\right\rangle:\left\{\psi_{j}\right\}\right)\right) & \text { for } j=1 \ldots n, \text { where } \mathcal{T}_{j} \text { comes } \\
& \text { from some } \mathbf{P} \text { inference in the } \\
& \text { derivation }
\end{array}
$$

Again, for $i=0 \ldots n$ we call $\mathcal{T}_{i}$ a tree from $\Gamma_{i} \vdash\left\langle m_{i}, s_{i}\right\rangle:\left\{\psi_{i}\right\}$, and we accompany the sequence of trees with a sequence $\Gamma_{0}, \Gamma_{1}, \ldots, \Gamma_{n}$, where $\Gamma_{i}=\operatorname{Gamma}\left(\mathcal{T}_{i}\right)$, for $i=0 \ldots n$. Together they represent a derivation from $\left\{x_{\left\langle\text {start }\left(s_{\text {init }}\right), s_{\text {init }}\right\rangle} \doteq 1\right\} \vdash\left\langle\operatorname{start}\left(s_{\text {init }}\right), s_{\text {init }}\right\rangle:\{\phi\}$ where $\Gamma_{\text {final }}=\Gamma^{0}$.

Unlike as in the soundness proof, these sequences are not given a priori. Indeed, we have to show they exist. We do this iteratively with the help of a couple of variables, collectively called the induction variables:

- The sequence of the $\mathcal{T}_{i}$ 's, initialized with a one-tree sequence $\mathcal{T}_{0}$ with a root node only labelled with $\left\{x_{\left\langle\operatorname{start}\left(s_{\text {init }}\right), s_{\text {init }}\right\rangle} \doteq 1\right\} \vdash\left\langle\operatorname{start}\left(s_{\text {init }}\right), s_{\text {init }}\right\rangle:\{\phi\}$ 
- The sequence of the $\Gamma_{i}$ 's initialized with a one-program sequence $\Gamma_{0}=\left\{x_{\left\langle\text {start }\left(s_{\text {init }}\right), s_{\text {init }}\right\rangle} \doteq 1\right\}$.

- A current substitution $\sigma$, initialized with

$$
\sigma=\left\{x_{\langle m, s\rangle}^{\alpha} \mapsto \operatorname{act}(m, s, \alpha) \mid\langle m, s\rangle \in M \times S \text { and } \alpha \in A(s)\right\} \cup\left\{x_{\left\langle\operatorname{start}\left(s_{\text {init }}\right), s_{\text {init }}\right\rangle}^{\{\phi} \mapsto 1\right\}
$$

To extend this initial state to a derivation we pick any leaf in any $\mathcal{T}_{i}$ and apply any inference rule to it, subject only to preference constraints. (This freedom is needed to match the claim that the inference rules can be applied in a don't-care fashion, subject only to the preference constraints.)

On applying an inference rule we will show that the following invariant is preserved: if $\Gamma \vdash\langle m, s\rangle: \Psi$ is the label of a node in some $\mathcal{T}_{i}$ then all of the following holds:

(i) $x_{\langle m, s\rangle}^{\psi} \in \operatorname{dom}(\sigma)$ and $\sigma$ is a solution of $\Gamma_{j}$, for all $j=0 \ldots n$.

(ii) $\operatorname{act}_{\sigma}(m, s, \alpha)=\operatorname{act}(m, s, \alpha)$ for every $\langle m, s\rangle \in M \times S$ such that $\langle m, s\rangle$ is in the policy-domain of $\Gamma_{0}$.

(iii) if $\Psi$ is a set of state formulas then

$$
\begin{aligned}
& \mathcal{M}_{\pi_{\text {fin }}(\sigma)},\langle m, s\rangle \mid=\Lambda \Psi \quad \text { iff } \quad \mathcal{M}_{\pi_{\text {fin }}},\langle m, s\rangle \models \bigwedge \Psi \quad \text { iff } \quad \sigma\left(x_{\langle m, s\rangle}^{\Psi}\right)=1 . \\
& \text { Moreover } \sigma\left(x_{\langle m, s\rangle}^{\Psi}\right) \in\{0,1\} .
\end{aligned}
$$

(iv) if $\Psi$ contains at least one proper path formula then

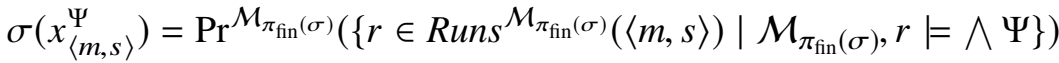

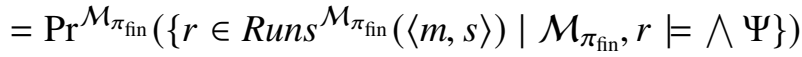

We refer to (i)-(iv) collectively as the invariant.

The invariant holds initially. This is trivial for each (i)-(iv). We will show below that the invariant is preserved by applying an inference rule, anywhere. This requires updating the induction variables appropriately. This process will end in the announced derivation, and, by construction, $\Gamma_{0}$ will be $\Gamma_{\text {final }}$.

Once this is done, the theorem is proved easily:

- That $\Gamma_{\text {final }}$ is satisfiable is trivial with (i).

- That $\operatorname{act}_{\sigma}(m, s, \alpha)=\operatorname{act}(m, s, \alpha)$ for every $m \times s$ in the policy-domain of $\Gamma_{\text {final }}$ becomes identical to (ii).

- $\mathcal{M}_{\pi_{\text {fin }}(\sigma)},\left\langle\operatorname{start}\left(s_{\text {init }}\right), s_{\text {init }}\right\rangle \mid=\phi$ follows from (iii) and $\mathcal{M}_{\pi_{\text {fin }}},\left\langle\operatorname{start}\left(s_{\text {init }}\right), s\right\rangle_{\text {init }}=\phi$ as given.

Hence it only remains to prove the invariant.

Choose some $\mathcal{T}_{i}$ and some leaf in $\mathcal{T}_{i}$ arbitrarily. Let $\Gamma \vdash\langle m, s\rangle: \Psi$ be the leaf's label. Assume the invariant holds for $\Gamma \vdash\langle m, s\rangle: \Psi$. Choose any inference rule applicable to $\Psi$, not violating preference constraints, and apply it. Then show that the invariant holds afterwards. 
In the first case $\Psi$ is a set of state formulas. Partition $\Psi=\{\phi\} \uplus \Psi^{\prime}$ to match the form of the inference rule.

If the rule is $\mathrm{T}$, add $\gamma_{\mathrm{one}}=x_{\langle m, s\rangle}^{\{\phi\} \uplus \Psi^{\prime}} \doteq x_{\langle m, s\rangle}^{\Psi^{\prime}}$ to $\Gamma_{i}$. All inference rules are mutually exclusive for a fixed selected formula $\phi$. Thus, if $\Gamma_{i}$, or any other $\Gamma_{j}$ for $i \neq j$, already contains an equality

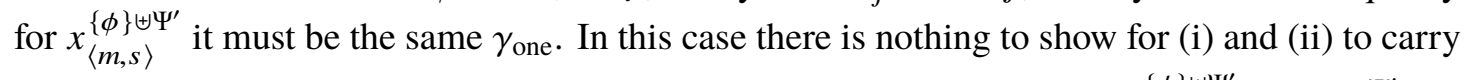
over. If, otherwise $\gamma_{\text {one }}$ is fresh then we extend $\sigma$ by the new binding $x_{\langle m, s\rangle}^{\{\phi\} \uplus \Psi^{\prime}} \mapsto \sigma\left(x_{\langle m, s\rangle}^{\Psi^{\prime}}\right)$. Because $x_{p}^{\{\phi\} \uplus \Psi^{\prime}}$ is fresh the new binding cannot interfere with those already in the domain of $\sigma$ before the binding was added. In any case

$$
\sigma\left(x_{\langle m, s\rangle}^{\{\phi\} \uplus \Psi^{\prime}}\right)=\sigma\left(x_{\langle m, s\rangle}^{\Psi^{\prime}}\right) .
$$

The applicability condition of T tells us $L(s) \mid=\phi$. With the semantics of PCTL* it follows

$$
\begin{gathered}
\mathcal{M}_{\pi_{\text {fin }}(\sigma)},\langle m, s\rangle \mid=\phi \wedge \wedge \Psi^{\prime} \text { iff } \mathcal{M}_{\pi_{\text {fin }}(\sigma)},\langle m, s\rangle \mid \wedge \Psi^{\prime} \\
\mathcal{M}_{\pi_{\text {fin }}},\langle m, s\rangle \mid=\phi \wedge \wedge \Psi^{\prime} \text { iff } \mathcal{M}_{\pi_{\text {fin }}},\langle m, s\rangle \mid \wedge \Psi^{\prime}
\end{gathered}
$$

Substituting (2), (3) and (4) into the iff-chain in (iii) gives

$$
\mathcal{M}_{\pi_{\text {fin }}},\langle m, s\rangle \mid=\wedge \Psi^{\prime} \quad \text { iff } \quad \mathcal{M}_{\pi_{\text {fin }}(\sigma)},\langle m, s\rangle=\wedge \Psi^{\prime} \quad \text { iff } \quad \sigma\left(x_{\langle m, s\rangle}^{\Psi^{\prime}}\right)=1 .
$$

which proves (iii) for the new leaf. For (iv) there is nothing to show. This concludes the proof for the case that the $T$ rule is applied.

The proofs for the inference rules $\boldsymbol{X}, \mathcal{J} \neg \neg, \neg \mathbf{P}, \mathbf{P} \neg, \wedge, \neg \wedge, \mathbf{P} 1, \mathbf{P} 2, \mathbf{P} 3$ all are analogous to the $T$ rule. We do not carry them out in detail. Notice again (as in the soundness proof) that in the $\neg \wedge$ rule the left and right conclusions are mutually exclusive, which enables correctly taking the sum of the corresponding variables.

It remains to consider the inference rules for $\mathbf{P}$-formulas. The rules are $\mathbf{Q}, \mathbf{P} T$ and $\mathbf{P X}$.

If $\mathbf{P}$ is applicable then there is a formula $\mathbf{P}_{\sim z} \phi \in \Psi$ such that $\gamma_{\text {left }} \notin \Gamma_{i}$ and $\gamma_{\text {right }} \notin \Gamma_{i}$.

The $\mathbf{P}$ inference starts a new derivation from $\emptyset \vdash\langle m, s\rangle:\{\phi\}$. In terms of our proof it starts with a tree $\mathcal{T}_{k}$ for $\emptyset \vdash\langle m, s\rangle:\{\phi\}$, for some $k>i$. The $\Gamma^{\prime}$ mentioned in the $\mathbf{P}$-rule is $\Gamma_{k}$ after that derivation has finished. Hence assume that this derivation (and possibly further sub-derivations) have already been carried out. We get as a result new trees starting from $\mathcal{T}_{k}$ and new programs starting from $\Gamma_{k}$ and the invariant will be preserved by induction. Property (i) then tells us that $\sigma$ is a solution of $\Gamma_{j}$, for all $j=0 \ldots n$. Now, with $\Gamma \subseteq \Gamma_{i}$ (by construction, we add all $\Gamma$ 's in the pivots as we encounter them) and the fact that in the $\mathbf{P}$ inference the program $\Gamma^{\prime}$ is just $\Gamma_{k}$ it follows that $\sigma$ is a solution for $\Gamma \cup \Gamma^{\prime}$ and we could add $\Gamma \cup \Gamma^{\prime}$ to $\Gamma_{i}$ without affecting (i).

The conclusion of the P-inference, however, is either $\Gamma \cup \Gamma^{\prime} \cup\left\{\gamma_{\text {left }}\right\}$ or $\Gamma \cup \Gamma^{\prime}\left\{\gamma_{\text {right }}\right\}$. Notice we already have the tree $\mathcal{T}_{k}$ for $\emptyset \vdash\langle m, s\rangle:\{\phi\}$. By invariant (iv) $\sigma$ will contain a

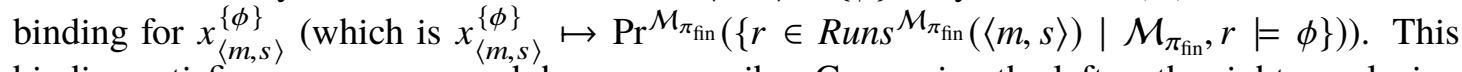
binding satisfies $\gamma_{\text {left }}$ or $\gamma_{\text {right }}$ and, hence, prescribes Chooseing the left or the right conclusion for extending $\mathcal{T}_{i}$ so that invariant (i) is preserved. Properties (ii), (iii) and (iv) are all trivially preserved by the extension.

The open cases are the Tand the $\mathbf{P} \boldsymbol{X}$ rules. Their proofs are analogous to the Tand the $\boldsymbol{X}$ rules and are omitted. 
This completes the proof for the case that $\Psi$ is a set of state formulas. Hence assume now that $\Psi$ contains at least one state formula. The relevant invariant properties are (i), (ii) and (iv).

The proofs are analogous to the ones when $\Psi$ is a set of state formulas. Essentially, the invariant follows from the design of the inference rules. This holds true also for the $\mathbf{X}$-rule, which is a bit tedious to inspect. One has to verify that it correctly reflects the transition probability function of the Markov chain $\mathcal{M}_{\pi_{\text {fin }}}$, cf. Section 2. Two important issues, though.

First, the $\mathrm{A}$ inferences are preceding the $\mathbf{X}$-inferences. Unlike as in the soundness proof, the $A$ inferences must be carried out consistently with the given policy $\pi_{\text {fin }}$. More precisely, we Choose the left conclusion $x_{\langle m, s\rangle}^{\alpha} \doteq 0$ in an A inference if act $(m, s, \alpha)=0$, and we CHoose the right conclusion $x_{\langle m, s\rangle}^{\alpha}>0$ otherwise. The variable $x_{\langle m, s\rangle}^{\alpha}$ is already in the domain of $\sigma$ because a binding to the value $x_{\langle m, s\rangle}^{\alpha} \mapsto \operatorname{act}(m, s, \alpha)$ was put there initially. This shows that the invariant is preserved for $\mathrm{A}$ inferences.

At the end of the derivation all bindings for the variables $x_{\langle m, s\rangle}^{\alpha}$ such that $\langle m, s\rangle$ is not in the policy domain of any $\mathcal{T}_{j}$ can be removed from $\sigma$. This allows us to prove the missing detail of invariant (ii).

Second, BSCCs and Forceing. Suppose the node we are looking at is $u$, the one with the sequent $\Gamma \vdash\langle m, s\rangle: \Psi$. The derivation adds a constraint $x_{\langle m, s\rangle}^{\psi} \doteq \chi$ for some $\chi \in\{0,1\}$ if $u$ is the root of a BSCC in $\mathcal{T}_{i}$. We need to argue for the correctness of doing that.

That $u$ is the root of a BSCC has a meaning in terms of the Markov Chain induced by the MDP $\mathcal{M}$ and the policy $\pi_{\text {fin }}$ : the policy state $\langle m, s\rangle$ is an entry node into a BSCC in the state transition diagram of this Markov Chain. The state transitions are given by the actions prescribed by $\pi_{\text {fin }}$, and the same actions are prescribed for the state transitions in the BSCC rooted at $u$. This follows from the A inferences as just explained. This is why the runs from $\langle m, s\rangle$ in the Markov Chain are exactly the same as the runs from $\langle m, s\rangle$ starting from $u$ in $\mathcal{T}_{i}$ by going down branches and following backlinks. Because in BSCCs in Markov Chains either all (fair) runs or no run

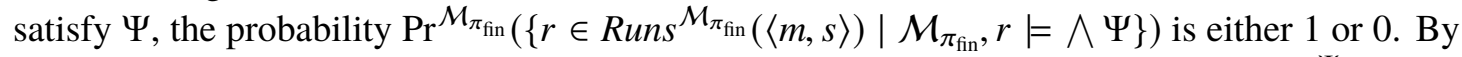
the correctness of ForCEing (cf. the soundness proof) the corresponding constraint $x_{\langle m, s\rangle}^{\Psi} \doteq 1$ or $x_{\langle m, s\rangle}^{\Psi} \doteq 0$ will be correctly added in $\Gamma_{i}$. This is sufficient to prove the invariant (iv).

\section{References}

[1] E. Altman. Constrained Markov Decision Processes, Volume 7. CRC Press, 1999.

[2] C. Baier, M. Größer, M. Leucker, B Bollig, and F. Ciesinski. Controller Synthesis for Probabilistic Systems. In TCS2004, 2004.

[3] C. Baier and J. Katoen. Principles of Model Checking. MIT Press, 2008.

[4] T. Brázdil, V. Brozek, V. Forejt, and A. Kucera. Stochastic Games With Branching-time Winning Objectives. In 21th IEEE Symp. on Logic in Computer Science LICS, 2006.

[5] T. Brázdil and V. Forejt. Strategy Synthesis for Markov Decision Processes and Branchingtime Logics. In 18th Int. Conf. on Concurrency Theory CONCUR, 2007. 
[6] T. Brázdil, V. Forejt, and A. Kucera. Controller Synthesis and Verification for Markov Decision Processes With Qualitative Branching Time Objectives. In ICALP, 2008.

[7] T. Brázdil, A. Kucera, and O. Strazovský. On the Decidability of Temporal Properties of Probabilistic Pushdown Automata. In STACS, 2005.

[8] C. Courcoubetis and M. Yannakakis. The Complexity of Probabilistic Verification. J. ACM, 42(4):857-907, 1995.

[9] X. C. Ding, A. Pinto, and A. Surana. Strategic Planning Under Uncertainties via Constrained Markov Decision Processes. In IEEE Int. Conf. on Robotics and Automation ICRA, 2013.

[10] X. C. Ding, S. Smith, C. Belta, and D. Rus. Optimal Control of Markov Decision Processes With Linear Temporal Logic Constraints. IEEE Trans. Automat. Contr., 59(5):1244-1257, 2014.

[11] D. Dolgov and E. Durfee. Stationary Deterministic Policies for Constrained Mdps With Multiple Rewards, Costs, and Discount Factors. In IJCAI, 2005.

[12] V. Forejt, M. Z. Kwiatkowska, G. Norman, and D. Parker. Automated Verification Techniques for Probabilistic Systems. In SFM, 2011.

[13] J. Kemeny, J. Snell, and A. Knapp. Denumerable Markov Chains: With a Chapter of Markov Random Fields by David Griffeath, volume 40. Springer, 2012.

[14] A. Kucera and O. Strazovský. On the Controller Synthesis for Finite-state Markov Decision Processes. In Theoretical Computer Science, 2005.

[15] M. Z. Kwiatkowska, G. Norman, and D. Parker. Stochastic Model Checking. In SFM, 2007.

[16] M. Z. Kwiatkowska and D. Parker. Automated Verification and Strategy Synthesis for Probabilistic Systems. In ATVA, 2013.

[17] M. Reynolds. A New Rule for LTL Tableaux. In GandALF, 2016.

[18] M. Reynolds. A Traditional Tree-style Tableau for LTL. CoRR, abs/1604.03962, 2016.

[19] J. Sprauel, A. Kolobov, and F. Teichteil-Königsbuch. Saturated Path-constrained MDP: Planning Under Uncertainty and Deterministic Model-checking Constraints. In AAAI, 2014.

[20] M. Svorenová, I. Cerna, and C. Belta. Optimal Control of Mdps With Temporal Logic Constraints. In $C D C, 2013$.

[21] F. Trevizan, S. Thiébaux, P. Santana, and B. Williams. Heuristic Search in Dual Space for Constrained Stochastic Shortest Path Problems. In ICAPS, 2016. 